\title{
Analysis of a Coupled System of Nonlinear Fractional Langevin Equations with Certain Nonlocal and Nonseparated Boundary Conditions
}

\author{
Zaid Laadjal $\mathbb{D}^{1},{ }^{1}$ Qasem M. Al-Mdallal ${ }^{\mathbb{D}},{ }^{2}$ and Fahd Jarad $\mathbb{D}^{3,4}$ \\ ${ }^{1}$ Department of Mathematics and Computer Sciences, ICOSI Laboratory, Abbes Laghrour University, Khenchela 40004, Algeria \\ ${ }^{2}$ Department of Mathematical Sciences, United Arab Emirates University, P. O. Box 15551, Al Ain, Abu Dhabi, UAE \\ ${ }^{3}$ Department of Mathematics, Çankaya University, Etimesgut, Ankara, Turkey \\ ${ }^{4}$ Department of Medical Research, China Medical University, Taichung 40402, Taiwan
}

Correspondence should be addressed to Qasem M. Al-Mdallal; q.almdallal@uaeu.ac.ae and Fahd Jarad; fahd@cankaya.edu.tr

Received 11 June 2021; Revised 28 August 2021; Accepted 3 September 2021; Published 24 September 2021

Academic Editor: Xiangfeng Yang

Copyright ( $\odot 2021$ Zaid Laadjal et al. This is an open access article distributed under the Creative Commons Attribution License, which permits unrestricted use, distribution, and reproduction in any medium, provided the original work is properly cited.

In this article, we use some fixed point theorems to discuss the existence and uniqueness of solutions to a coupled system of a nonlinear Langevin differential equation which involves Caputo fractional derivatives of different orders and is governed by new type of nonlocal and nonseparated boundary conditions consisting of fractional integrals and derivatives. The considered boundary conditions are totally dissimilar than the ones already handled in the literature. Additionally, we modify the Adams-type predictor-corrector method by implicitly implementing the Gauss-Seidel method in order to solve some specific particular cases of the system.

\section{Introduction}

The fractional calculus is the ramification of mathematics concerning the integrals and derivatives of functions with arbitrary orders. It has a long history that goes back to more than three hundred years. Nonetheless, researchers discovered the importance and effectiveness of this calculus just a mere in the last few decades. It turned out that the fractional integrals and derivatives are very good tools in modeling some phenomena. This was concluded simply because of the amazing results obtained when some of the researchers used the implements in the fractional calculus for the sake of understanding real world problems happening in the environment surrounding. Recently, differential equations of fractional order have been applied in various fields like physical, biology, chemistry, control theory, electrical circuits, blood flow phenomena, and signal and image processing; for more details, see [1-3] and references cited therein.
In 1908, Langevin [4] formulated his famous equation containing derivative of integer order. This equation describes the evolution of certain physical phenomena in fluctuating environments [5]. The Langevin equation was used in large part to describe some phenomena such as anomalous transport [6]. The Langevin equation has been recently extended to the fractional order by Lim et al. [7]. They acquainted a new form of Langevin equations involving two different fractional order for the sake of describing the viscoelastic anomalous diffusion in the complex liquids. We refer the reader to Subsection 2.1 in [3] and the references cited therein for further details. Uranagase and Munakata [8] discussed the generalized Langevin equation with emphasis on a mechanical random force whose time evolution is not natural due to the presence of a projection operator in a propagator. Lozinski et al. [9] discussed the applications of Langevin and Fokker-Planck equations in polymer rheology and stochastic simulation techniques for solving this equation. Laadjal et al. [10] presented the 
existence and uniqueness of solutions for the multiterm fractional Langevin equation with boundary conditions.

Using the tools in mathematical analysis and the theory of fixed points, discussing the qualitative specification encapsuling the behaviors of solutions of differential equations in fractional derivatives settings has attracted the attention of many scientists. To get an update about the works in the literature, we ask the readers to investigate [11] and the references cited there. On the top of this, classes of systems of fractional differential equations with separated (or nonseparated) boundary conditions have been studied intensively in literatures [12-14].

Motivated by what are mentioned above and the recent development on Langevin equations, in this paper, we discuss the existence and the uniqueness of solutions to a coupled system of fractional Langevin equations in the form as follows:

$$
\begin{cases}{ }^{c} D^{\alpha_{1}}\left({ }^{c} D^{\beta_{1}}+\lambda\right) \psi_{1}(t)=f\left(t, \psi_{1}(t), \psi_{2}(t)\right), & t \in J, 0<\alpha_{1} \leq 1<\beta_{1} \leq 2, \\ { }^{c} D^{\alpha_{2}}\left({ }^{c} D^{\beta_{2}}+k\right) \psi_{2}(t)=g\left(t, \psi_{1}(t), \psi_{2}(t)\right), & t \in J, 0<\alpha_{2} \leq 1<\beta_{2} \leq 2,\end{cases}
$$

subject to a new type of nonlocal nonseparated boundary conditions as follows:

$$
\left\{\begin{array}{l}
\psi_{1}(0)=a_{0}, \psi_{2}(0)=b_{0}, \psi_{1}^{\prime}(0)=\psi_{2}^{\prime}(0)=0, \\
\psi_{1}(\xi)=a\left({ }^{c} D^{p} \psi_{2}\right)\left(\mu_{1}\right), \xi \in(0,1], \mu_{1} \in J, 0<p<\beta_{2}, \\
\psi_{2}(\eta)=b\left(I^{q} \psi_{1}\right]\left(\mu_{2}\right], \eta \in(0,1], \mu_{2} \in J, q \geq 0,
\end{array}\right.
$$

where $J=[0,1], \lambda, k, a_{0}, b_{0}, a, b$ are real constants, ${ }^{c} D^{i}$ where $i=\alpha_{1}, \beta_{1}, \alpha_{2}, \beta_{2}, p$ are the Caputo fractional derivatives of order $\alpha_{1}, \beta_{1}, \alpha_{2}, \beta_{2}$, and $p$, respectively, $f, g: J \times \mathbb{R} \times$ $\mathbb{R} \longrightarrow \mathbb{R}$ are given functions, and $I^{q}$ is the Riemann-Liouville fractional integral of order $q$. By using the Banach contraction principle and Leray-Schauder alternative fixed point theorem, we investigate the existence of solutions for problems (1) and (2). We remark that the boundary value problem discussed here is distinctive of the ones discussed in literatures [12-14].

This article is organized as follows. In Section 2, we present some definitions, theorems, and related lemmas used in next sections. Section 3 discusses the existence and uniqueness of the system under consideration. In Section 4, we furnish some numerical examples. Section 5 is devoted to our concluding remarks.

\section{Preliminaries}

Definition 1. (see $[1,2])$. Let $a, b \in \mathbb{R}(-\infty<a<b<\infty)$. The Riemann-Liouville fractional integral of order $\alpha \in \mathbb{R}^{+}$for a function $f \in L^{1}[a, b]$ is defined by

$$
I_{a}^{\alpha} f(t)=\frac{1}{\Gamma(\alpha)} \int_{a}^{t}(t-s)^{\alpha-1} f(s) \mathrm{d} s, \quad \text { for } \alpha>0, \text { and } I_{a}^{0} f(t)=f(t)
$$

where $\Gamma$ is the Euler Gamma function.

Definition 2 (see $[1,2]$ ). The Caputo fractional derivative of order $\alpha \in \mathbb{R}^{+}$for a function $f \in C^{n}[a, b]$ is defined by

$$
{ }^{c} D_{a}^{\alpha} f(t)=I_{a}^{n-\alpha} D^{n} f(t), \quad \text { for } \alpha>0 \text {, and }{ }^{c} D_{a}^{0} f(t)=f(t),
$$

where $n-1<\alpha \leq n, n \in \mathbb{N}$, and $D^{n}=\mathrm{d}^{n} / \mathrm{d} t^{n}$.

Proposition 3 (see [2]). For $\beta>0$ and $\alpha>0$ and $f \in L^{1}[a, b]$, we have the following properties:

$$
\begin{aligned}
I_{a}^{\alpha} I_{a}^{\beta} f(t) & =I_{a}^{\beta} I_{a}^{\alpha} f(t)=I_{a}^{\alpha+\beta} f(t) . \\
I_{a}^{\alpha}(t-a)^{\mu} & =\frac{\Gamma(\mu+1)}{\Gamma(\alpha+\mu+1)}(t-a)^{\alpha+\mu}, \mu>-1 . \\
{ }^{c} D_{a}^{\alpha}\left(I_{a}^{\beta} f(t)\right) & =I_{a}^{\beta-\alpha} f(t), \quad(\text { here } \beta \geq \alpha>0) .
\end{aligned}
$$

Proposition 4 (see [2]). Let $\alpha>0$ with $n-1<\alpha \leq n$ and $f \in C^{n}[a, b]$. Then,

$$
I_{a}^{\alpha}\left[{ }^{c} D_{a}^{\alpha} f(t)\right]=f(t)-\sum_{k=0}^{n-1} c_{k}(t-a)^{k},
$$

where $c_{k}=f^{(k)}(a) / k$ !. In particular, when $0<\alpha \leq 1$, we have

$$
I_{0}^{\alpha}\left[{ }^{c} D_{0}^{\alpha} f(t)\right]=f(t)-f(0)
$$

Proposition 5 (see [2]). Let $\mu>0$ and $\alpha>0$ with $n-1<\alpha \leq n$. Then,

$$
\begin{aligned}
{ }^{c} D_{a}^{\alpha}(t-a)^{\mu-1} & =\frac{\Gamma(\mu)}{\Gamma(\mu-\alpha)}(t-a)^{\mu-\alpha-1}, \mu>\mathrm{n}, \\
{ }^{c} D_{a}^{\alpha}(t-a)^{k} & =0, k=0,1, \ldots, n-1 .
\end{aligned}
$$

Theorem 6 (Leray-Schauder alternative [15]). Let $T: X \longrightarrow X$ be a completely continuous operator (i.e., a map that is restricted to any bounded set in $X$ is compact). Let $M(T)=\{u \in X: u=m T(u)$ forsome $0<m<1\}$. Then, either the set $M(T)$ is unbounded or $T$ has at least one fixed point.

For the sake of simplicity, henceforwards we will write $I^{\alpha}$ and ${ }^{c} D^{\alpha}$ instead of $I_{0}^{\alpha}$ and ${ }^{c} D_{0}^{\alpha}$, respectively. 


\section{Main Results}

In this section, we will discuss the existence and uniqueness of the solution to systems (1)-(2).

Lemma 7. Let $\quad v, w \in C([0,1], \mathbb{R}), \quad 0<\alpha_{1}, \alpha_{2} \leq 1$, $1<\beta_{1}, \beta_{2} \leq 2, q \geq 0,0<p<\beta_{2}, \quad\left(\bar{\alpha}_{1}:=\alpha_{1}+\beta_{1}+q, \bar{\alpha}_{2}:=\alpha_{2}\right.$ $\left.+\beta_{2}-p\right)$, and

$$
\Delta=\frac{\xi^{\beta_{1}} \eta^{\beta_{2}}}{\Gamma\left(\beta_{1}+1\right) \Gamma\left(\beta_{2}+1\right)}-\frac{a b \mu_{1}^{\beta_{2}-p} \mu_{2}^{\beta_{1}+q}}{\Gamma\left(\beta_{1}+q+1\right) \Gamma\left(\beta_{2}-p+1\right)} \neq 0 .
$$

Then, the solution of the following coupled system of fractional Langevin equations is as follows:

$$
\begin{aligned}
& { }^{c} D^{\alpha_{1}}\left({ }^{c} D^{\beta_{1}}+\lambda\right) \psi_{1}(t)=v(t), \\
& { }^{c} D^{\alpha_{2}}\left({ }^{c} D^{\beta_{2}}+k\right) \psi_{2}(t)=w(t),
\end{aligned}
$$

equipped with the boundary condition (2) which is equivalent to the coupled system of the following integral equations:

$$
\begin{aligned}
\psi_{1}(t)= & I^{\alpha_{1}+\beta_{1}} v(t)-\lambda I^{\beta_{1}} \psi_{1}(t)+t^{\beta_{1}}\left\{A_{1}\left[a I^{\bar{\alpha}_{2}} w\left(\mu_{1}\right)-a k I^{\beta_{2}-p} \psi_{2}\left(\mu_{1}\right)-I^{\alpha_{1}+\beta_{1}} v(\xi)+\lambda I^{\beta_{1}} \psi_{1}(\xi)\right]\right. \\
& \left.+A_{2}\left[b I^{\bar{\alpha}_{1}} v\left(\mu_{2}\right)-b \lambda I^{\beta_{1}+q} \psi_{1}\left(\mu_{2}\right)-I^{\alpha_{2}+\beta_{2}} w(\eta)+k I^{\beta_{2}} \psi_{2}(\eta)\right]+A_{3}\right\}+a_{0}, \\
\psi_{2}(t)= & I^{\alpha_{2}+\beta_{2}} w(t)-k I^{\beta_{2}} \psi_{2}(t)+t^{\beta_{2}}\left\{B_{1}\left[b I^{\bar{\alpha}_{1}} v\left(\mu_{2}\right)-b \lambda I^{\beta_{1}+q} \psi_{1}\left(\mu_{2}\right)-I^{\alpha_{2}+\beta_{2}} w(\eta)+k I^{\beta_{2}} \psi_{2}(\eta)\right]\right. \\
& \left.+B_{2}\left[a I^{\bar{\alpha}_{2}} w\left(\mu_{1}\right)-a k I^{\beta_{2}-p} \psi_{2}\left(\mu_{1}\right)-I^{\alpha_{1}+\beta_{1}} v(\xi)+\lambda I^{\beta_{1}} \psi_{1}(\xi)\right]+B_{3}\right\}+b_{0},
\end{aligned}
$$

where

$$
\begin{aligned}
& A_{1}=\frac{1}{\Delta \Gamma\left(\beta_{1}+1\right)} \frac{\eta^{\beta_{2}}}{\Gamma\left(\beta_{2}+1\right)}, \\
& A_{2}=\frac{1}{\Delta \Gamma\left(\beta_{1}+1\right)} \frac{a \mu_{1}^{\beta_{2}-p}}{\Gamma\left(\beta_{2}-p+1\right)}, \\
& A_{3}=\frac{1}{\Delta \Gamma\left(\beta_{1}+1\right)}\left[-\frac{a_{0} \eta^{\beta_{2}}}{\Gamma\left(\beta_{2}+1\right)}+\frac{a \mu_{1}^{\beta_{2}-p}}{\Gamma\left(\beta_{2}-p+1\right)}\left(\frac{b a_{0} \mu_{2}^{q}}{\Gamma(q+1)}-b_{0}\right)\right], \\
& B_{1}=\frac{1}{\Delta \Gamma\left(\beta_{2}+1\right)} \frac{\xi^{\beta_{1}}}{\Gamma\left(\beta_{1}+1\right)}, \\
& B_{2}=\frac{1}{\Delta \Gamma\left(\beta_{2}+1\right)} \frac{b \mu_{2}^{q+\beta_{1}}}{\Gamma\left(q+\beta_{1}+1\right)}, \\
& B_{3}=\frac{1}{\Delta \Gamma\left(\beta_{2}+1\right)}\left[-\frac{b a_{0} \mu_{2}^{q+\beta_{1}}}{\Gamma\left(q+\beta_{1}+1\right)}+\frac{\xi^{\beta_{1}}}{\Gamma\left(\beta_{1}+1\right)}\left(\frac{b a_{0} \mu_{2}^{q}}{\Gamma(q+1)}-b_{0}\right)\right] .
\end{aligned}
$$

Proof. Applying the operator $I^{\alpha_{1}+\beta_{1}}$ and $I^{\alpha_{2}+\beta_{2}}$ on (10) and

(11), respectively, we get 


$$
\begin{aligned}
& \psi_{1}(t)=I^{\alpha_{1}+\beta_{1}} v(t)-\lambda I^{\beta_{1}} \psi_{1}(t)+\frac{c_{2} t^{\beta_{1}}}{\Gamma\left(\beta_{1}+1\right)}+c_{1} t+c_{0} \\
& \psi_{2}(t)=I^{\alpha_{2}+\beta_{2}} w(t)-k I^{\beta_{2}} \psi_{2}(t)+\frac{\bar{c}_{2} t^{\beta_{2}}}{\Gamma\left(\beta_{2}+1\right)}+\bar{c}_{1} t+\bar{c}_{0}
\end{aligned}
$$

where $c_{i}, \bar{c}_{i} \in \mathbb{R}(i=0,1,2)$. From the boundary conditions $\psi_{1}(0)=a_{0}, \psi_{2}(0)=b_{0}$, and $\psi_{1}^{\prime}(0)=\psi_{2}^{\prime}(0)=0$, we get $c_{0}=a_{0}, \bar{c}_{0}=b_{0}$, and $c_{1}=\bar{c}_{1}=0$, respectively. Next, using the nonlocal integral conditions $\psi_{1}(\xi)=a\left({ }^{c} D^{p} \psi_{2}\right)\left(\mu_{1}\right)$ and $\psi_{2}(\eta)=b\left(I^{q} \psi_{1}\right)\left(\mu_{2}\right)$, we obtain that

$$
\begin{gathered}
I^{\alpha_{1}+\beta_{1}} v(\xi)-\lambda I^{\beta_{1}} \psi_{1}(\xi)+\frac{c_{2} \xi^{\beta_{1}}}{\Gamma\left(\beta_{1}+1\right)}+a_{0}=a I^{\bar{\alpha}_{2}} w\left(\mu_{1}\right) \\
-a k I^{\beta_{2}-p} \psi_{2}\left(\mu_{1}\right)+\frac{a \bar{c}_{2} \mu_{1}^{\beta_{2}-p}}{\Gamma\left(\beta_{2}-p+1\right)},
\end{gathered}
$$

$$
\begin{aligned}
I^{\alpha_{2}+\beta_{2}} w(\eta) & -k I^{\beta_{2}} \psi_{2}(\eta)+\frac{\bar{c}_{2} \eta^{\beta_{2}}}{\Gamma\left(\beta_{2}+1\right)}+b_{0}=b I^{\bar{\alpha}_{1}} v\left(\mu_{2}\right) \\
& -b \lambda I^{\beta_{1}+q} \psi_{1}\left(\mu_{2}\right)+\frac{c_{2} b \mu_{2}^{\beta_{1}+q}}{\Gamma\left(\beta_{1}+q+1\right)}+\frac{b a_{0} \mu_{2}^{q}}{\Gamma(q+1)}
\end{aligned}
$$

Solving the above system, we find that

$$
\begin{aligned}
c_{2}= & \frac{1}{\Delta}\left[\frac{\eta^{\beta_{2}}}{\Gamma\left(\beta_{2}+1\right)}\left[a I^{\bar{\alpha}_{2}} w\left(\mu_{1}\right)-a k I^{\beta_{2}-p} \psi_{2}\left(\mu_{1}\right)-I^{\alpha_{1}+\beta_{1}} v(\xi)+\lambda I^{\beta_{1}} \psi_{1}(\xi)\right]\right. \\
& +\frac{a \mu_{1}^{\beta_{2}-p}}{\Gamma\left(\beta_{2}+1-p\right)}\left[b I^{\bar{\alpha}_{1}} v\left(\mu_{2}\right)-b \lambda I^{\beta_{1}+q} \psi_{1}\left(\mu_{2}\right)-I^{\alpha_{2}+\beta_{2}} w(\eta)+k I^{\beta_{2}} \psi_{2}(\eta)\right] \\
& \left.-\frac{a_{0} \eta^{\beta_{2}}}{\Gamma\left(\beta_{2}+1\right)}+\frac{a \mu_{1}^{\beta_{2}-p}}{\Gamma\left(\beta_{2}-p+1\right)}\left(\frac{b a_{0} \mu_{2}^{q}}{\Gamma(q+1)}-b_{0}\right)\right] \\
\bar{c}_{2}= & \frac{1}{\Delta}\left[\frac{\xi^{\beta_{1}}}{\Gamma\left(\beta_{1}+1\right)}\left[b I^{\bar{\alpha}_{1}} v\left(\mu_{2}\right)-b \lambda I^{\beta_{1}+q} \psi_{1}\left(\mu_{2}\right)-I^{\alpha_{2}+\beta_{2}} w(\eta)+k I^{\beta_{2}} \psi_{2}(\eta)\right]\right. \\
& +\frac{b \mu_{2}^{q+\beta_{1}}}{\Gamma\left(q+\beta_{1}+1\right)}\left[a I^{\bar{\alpha}_{2}} w\left(\mu_{1}\right)-a k I^{\beta_{2}-p} \psi_{2}\left(\mu_{1}\right)-I^{\alpha_{1}+\beta_{1}} v(\xi)+\lambda I^{\beta_{1}} \psi_{1}(\xi)\right] \\
& \left.-\frac{a_{0} b \mu_{2}^{q+\beta_{1}}}{\Gamma\left(q+\beta_{1}+1\right)}+\frac{\xi^{\beta_{1}}}{\Gamma\left(\beta_{1}+1\right)}\left(\frac{b a_{0} \mu_{2}^{q}}{\Gamma(q+1)}-b_{0}\right)\right],
\end{aligned}
$$

where $\Delta$ is the determinant of the matrix associated with systems (17)-(18) in the two variables $c_{2}$ and $\bar{c}_{2}$, and it is given by (9).

Substituting the values of $c_{0}, \bar{c}_{0}, c_{1}, \bar{c}_{1}, c_{2}$, and $\bar{c}_{2}$ in (15) and (16), we obtain the system of the integral equations (12) and (13). The proof is completed.
The Banach space $E=C([0,1], \mathbb{R})$ is defined with the norm $\left\|\psi_{1}\right\|_{E}=\sup _{t \in J}\left|\psi_{1}(t)\right|$.

So, the space $E \times E=\left\{\left(\psi_{1}, \psi_{2}\right)\right.$, s.t $\left.\left(\psi_{1}, \psi_{2}\right) \in E \times E\right\}$ with the norm $\left\|\left(\psi_{1}, \psi_{2}\right)\right\|_{E \times E}=\left\|\psi_{1}\right\|_{E}+\left\|\psi_{2}\right\|_{E}$ is Banach space.

Now, let us define the operator $\mathfrak{N}: E \times E \longrightarrow E \times E$, by $\mathfrak{N}\left(\psi_{1}, \psi_{2}\right)(t)=\left(\mathfrak{N}_{1}\left(\psi_{1}, \psi_{2}\right)(t), \mathfrak{N}_{2}\left(\psi_{1}, \psi_{2}\right)(t)\right)$, where 


$$
\begin{aligned}
& \mathfrak{N}_{1}\left(\psi_{1}, \psi_{2}\right)(t)=\frac{1}{\Gamma\left(\alpha_{1}+\beta_{1}\right)} \int_{0}^{t}(t-s)^{\alpha_{1}+\beta_{1}-1} f\left(s, \psi_{1}(s), \psi_{2}(s)\right) \mathrm{d} s-\frac{\lambda}{\Gamma\left(\beta_{1}\right)} \int_{0}^{t}(t-s)^{\beta_{1}-1} \psi_{1}(s) \mathrm{d} s \\
& +t^{\beta_{1}}\left\{A _ { 1 } \left(\frac{a}{\Gamma\left(\bar{\alpha}_{2}\right)} \int_{0}^{\mu_{1}}\left(\mu_{1}-s\right)^{\bar{\alpha}_{2}-1} g\left(s, \psi_{1}(s), \psi_{2}(s)\right) \mathrm{d} s-\frac{a k}{\Gamma\left(\beta_{2}-p\right)}\right.\right. \\
& \times \int_{0}^{\mu_{1}}\left(\mu_{1}-s\right)^{\beta_{2}-p-1} \psi_{2}(s) \mathrm{d} s-\frac{1}{\Gamma\left(\alpha_{1}+\beta_{1}\right)} \int_{0}^{\xi}(\xi-s)^{\alpha_{1}+\beta_{1}-1} f\left(s, \psi_{1}(s), \psi_{2}(s)\right) \mathrm{d} s \\
& \left.+\frac{\lambda}{\Gamma\left(\beta_{1}\right)} \int_{0}^{\xi}(\xi-s)^{\beta_{1}-1} \psi_{1}(s) \mathrm{d} s\right)+A_{2}\left(\frac{b}{\Gamma\left(\bar{\alpha}_{1}\right)} \int_{0}^{\mu_{2}}\left(\mu_{2}-s\right)^{\bar{\alpha}_{1}-1} f\left(s, \psi_{1}(s), \psi_{2}(s)\right) \mathrm{d} s\right. \\
& -\frac{b \lambda}{\Gamma\left(\beta_{1}+q\right)} \int_{0}^{\mu_{2}}\left(\mu_{2}-s\right)^{\beta_{1}+q-1} \psi_{1}(s) \mathrm{d} s-\frac{1}{\Gamma\left(\alpha_{2}+\beta_{2}\right)} \int_{0}^{\eta}(\eta-s)^{\alpha_{2}+\beta_{2}-1} g\left(s, \psi_{1}(s), \psi_{2}(s)\right) \mathrm{d} s \\
& \left.\left.+\frac{k}{\Gamma\left(\beta_{2}\right)} \int_{0}^{\eta}(\eta-s)^{\beta_{2}-1} \psi_{2}(s) \mathrm{d} s\right)+A_{3}\right\}+a_{0} \\
& \mathfrak{N}_{2}\left(\psi_{1}, \psi_{2}\right)(t)=\frac{1}{\Gamma\left(\alpha_{2}+\beta_{2}\right)} \int_{0}^{t}(t-s)^{\alpha_{2}+\beta_{2}-1} g\left(s, \psi_{1}(s), \psi_{2}(s)\right) \mathrm{d} s-\frac{k}{\Gamma\left(\beta_{2}\right)} \int_{0}^{t}(t-s)^{\beta_{2}-1} \psi_{2}(s) \mathrm{d} s \\
& +t^{\beta_{2}}\left\{B _ { 1 } \left(\frac{b}{\Gamma\left(\bar{\alpha}_{1}\right)} \int_{0}^{\mu_{2}}\left(\mu_{2}-s\right)^{\bar{\alpha}_{1}-1} f\left(s, \psi_{1}(s), \psi_{2}(s)\right) \mathrm{d} s-\frac{b \lambda}{\Gamma\left(\beta_{1}+q\right)}\right.\right. \\
& \times \int_{0}^{\mu_{2}}\left(\mu_{2}-s\right)^{\beta_{1}+q-1} \psi_{1}(s) \mathrm{d} s-\frac{1}{\Gamma\left(\alpha_{2}+\beta_{2}\right)} \int_{0}^{\eta}(\eta-s)^{\alpha_{2}+\beta_{2}-1} g\left(s, \psi_{1}(s), \psi_{2}(s)\right) \mathrm{d} s \\
& +\frac{k}{\Gamma\left(\beta_{2}\right)} \int_{0}^{\eta}(\eta-s)^{\beta_{2}-1} \psi_{2}(s) \mathrm{d} s+B_{2}\left(\frac{a}{\Gamma\left(\bar{\alpha}_{2}\right)} \int_{0}^{\mu_{1}}\left(\mu_{1}-s\right)^{\bar{\alpha}_{2}-1} g\left(s, \psi_{1}(s), \psi_{2}(s)\right) \mathrm{d} s\right. \\
& -\frac{a k}{\Gamma\left(\beta_{2}-p\right)} \int_{0}^{\mu_{1}}\left(\mu_{1}-s\right)^{\beta_{2}-p-1} \psi_{2}(s) \mathrm{d} s-\frac{1}{\Gamma\left(\alpha_{1}+\beta_{1}\right)} \int_{0}^{\xi}(\xi-s)^{\alpha_{1}+\beta_{1}-1} f\left(s, \psi_{1}(s), \psi_{2}(s)\right) \mathrm{d} s \\
& \left.\left.+\frac{\lambda}{\Gamma\left(\beta_{1}\right)} \int_{0}^{\xi}(\xi-s)^{\beta_{1}-1} \psi_{1}(s) \mathrm{d} s\right)+B_{3}\right\}+b_{0} \text {. }
\end{aligned}
$$

Note that the couple $\left(\psi_{1}, \psi_{2}\right)$ is a fixed point of the operator $\mathfrak{N}$ if only if $\left(\psi_{1}, \psi_{2}\right)$ is a solution of systems (1)-(2). Consider the following hypotheses:

(H1). $f, g: J \times \mathbb{R} \times \mathbb{R} \longrightarrow \mathbb{R}$ are continuous functions, and there exist real positive constants $\sigma_{i}, \tau_{i}(i=0,1,2)$ such that

$$
\begin{aligned}
& |f(t, \zeta, \kappa)| \leq \sigma_{0}+\sigma_{1}|\zeta|+\sigma_{2}|\kappa|, \\
& |g(t, \zeta, \kappa)| \leq \tau_{0}+\tau_{1}|\zeta|+\tau_{2}|\kappa|,
\end{aligned}
$$

for all $(t, \zeta, \kappa) \in J \times \mathbb{R} \times \mathbb{R}$.

(H2). There exist constants $K, L>0$ such that

$$
\begin{gathered}
|f(t, \zeta, \kappa)-f(t, \bar{\zeta}, \bar{\kappa})| \leq K(|\zeta-\bar{\zeta}|+|\kappa-\bar{\kappa}|), \\
|g(t, \zeta, \kappa)-g(t, \bar{\zeta}, \bar{\kappa})| \leq L(|\zeta-\bar{\zeta}|+|\kappa-\bar{\kappa}|),
\end{gathered}
$$

for all $(t, \zeta, \kappa),(t, \bar{\zeta}, \bar{\kappa}) \in J \times \mathbb{R} \times \mathbb{R}$.

(H3). There exist $F_{0}, G_{0}>0$ such that $F_{0}=\sup _{t \in J}|f(t, 0,0)|$ and $G_{0}=\sup _{t \in J}|g(t, 0,0)|$.
Remark 8. From conditions (H1) and (H2) for all $\left(\psi_{1}, \psi_{2}\right) \in E \times E$, we get

$$
\begin{aligned}
& \left|f\left(t, \psi_{1}(t), \psi_{2}(t)\right)\right| \leq K\left\|\left(\psi_{1}, \psi_{2}\right)\right\|_{E \times E}+F_{0}, \\
& \left|g\left(t, \psi_{1}(t), \psi_{2}(t)\right)\right| \leq L\left\|\left(\psi_{1}, \psi_{2}\right)\right\|_{E \times E}+G_{0} .
\end{aligned}
$$

For computation convenience, we set the following constants:

$$
\begin{aligned}
& Q_{1}=\frac{1+\left|A_{1}\right| \xi^{\alpha_{1}+\beta_{1}}}{\Gamma\left(\alpha_{1}+\beta_{1}+1\right)}+\frac{\left|A_{2} b\right| \mu_{2}^{\bar{\alpha}_{1}}}{\Gamma\left(\bar{\alpha}_{1}+1\right)} \\
& Q_{2}=\frac{\left|a A_{1}\right| \mu_{1}^{\bar{\alpha}_{2}}}{\Gamma\left(\bar{\alpha}_{2}+1\right)}+\frac{\left|A_{2}\right| \eta^{\alpha_{2}+\beta_{2}}}{\Gamma\left(\alpha_{2}+\beta_{2}+1\right)}, \\
& Q_{3}=\frac{\left|b B_{1}\right| \mu_{2}^{\bar{\alpha}_{1}}}{\Gamma\left(\bar{\alpha}_{1}+1\right)}+\frac{\left|B_{2}\right| \xi^{\alpha_{1}+\beta_{1}}}{\Gamma\left(\alpha_{1}+\beta_{1}+1\right)},
\end{aligned}
$$




$$
\begin{aligned}
Q_{5} & =\frac{\left(1+\left|A_{1}\right| \xi^{\beta_{1}}\right)|\lambda|}{\Gamma\left(\beta_{1}+1\right)}+\frac{\left|A_{2}\right||b \lambda| \mu_{2}^{\beta_{1}+q}}{\Gamma\left(\beta_{1}+q+1\right)}, \\
Q_{6} & =\frac{\left|A_{1}\right||a k| \mu_{1}^{\beta_{2}-p}}{\Gamma\left(\beta_{2}-p+1\right)}+\frac{\left|A_{2}\right||k| \eta^{\beta_{2}}}{\Gamma\left(\beta_{2}+1\right)}, \\
Q_{7} & =\frac{\left|B_{2} \lambda\right| \xi^{\beta_{1}}}{\Gamma\left(\beta_{1}+1\right)}+\frac{\left|b \lambda B_{1}\right| \mu_{2}^{\beta_{1}+q}}{\Gamma\left(\beta_{1}+q+1\right)}, \\
Q_{8} & =\frac{\left|B_{2} a k\right| \mu_{1}^{\beta_{2}-p}}{\Gamma\left(\beta_{2}-p+1\right)}+\frac{\left(1+\left|B_{1}\right| \eta^{\beta_{2}}\right)|k|}{\Gamma\left(\beta_{2}+1\right)}, \\
\rho_{1} & =\max \left\{Q_{5}, Q_{6}\right\}, \\
\rho_{2} & =\max \left\{Q_{7}, Q_{8}\right\} .
\end{aligned}
$$

In the following step, we present the following result about the uniqueness of solutions for problems (1)-(2) by applying the Banach contraction principle.
Theorem 9. Assume that $(\mathrm{H} 2)$ and $(\mathrm{H} 3)$ hold. If

$$
\left[\left(Q_{1}+Q_{3}\right) K+\left(Q_{2}+Q_{4}\right) L+\rho_{1}+\rho_{2}\right]<1,
$$

where $Q_{1}, Q_{2}, Q_{3}$, and $Q_{4}$ and $\rho_{1}$ and $\rho_{2}$ are, respectively, defined by (24), (25), and (28), then the boundary value problems (1)-(2) have a unique solution.

Proof. Choose a positive real constant $R$ where

$$
R \geq \frac{\left(Q_{1}+Q_{3}\right) F_{0}+\left(Q_{2}+Q_{4}\right) G_{0}+\left|A_{3}\right|+\left|B_{3}\right|+\left|a_{0}\right|+\left|b_{0}\right|}{1-\left[\left(Q_{1}+Q_{3}\right) K+\left(Q_{2}+Q_{4}\right) L+\rho_{1}+\rho_{2}\right]} .
$$

Let $B_{R}=\left\{\left(\psi_{1}, \psi_{2}\right) \in E \times\right.$ Es.t. $\left.\left\|\left(\psi_{1}, \psi_{2}\right)\right\|_{E \times E} \leq R\right\}$. First, we prove that $\mathfrak{N}\left(B_{R}\right) \subseteq B_{R}$.

For all $\left(\psi_{1}, \psi_{2}\right) \in B_{R}$, we have

$$
\begin{aligned}
& \left|\mathfrak{N}_{1}\left(\psi_{1}, \psi_{2}\right)(t)\right| \leq \frac{K R+F_{0}}{\Gamma\left(\alpha_{1}+\beta_{1}\right)} \int_{0}^{t}(t-s)^{\alpha_{1}+\beta_{1}-1} \mathrm{~d} s+\frac{|\lambda|\left\|\psi_{1}\right\|_{E}}{\Gamma\left(\beta_{1}\right)} \int_{0}^{t}(t-s)^{\beta_{1}-1} \mathrm{~d} s \\
& +t^{\beta_{1}}\left\{| A _ { 1 } | \left(\frac{|a|\left(L R+G_{0}\right)}{\Gamma\left(\bar{\alpha}_{2}\right)} \int_{0}^{\mu_{1}}\left(\mu_{1}-s\right)^{\bar{\alpha}_{2}-1} \mathrm{~d} s+\frac{|a k|\left\|\psi_{2}\right\|_{E}}{\Gamma\left(\beta_{2}-p\right)} \int_{0}^{\mu_{1}}\left(\mu_{1}-s\right)^{\beta_{2}-p-1} \mathrm{~d} s\right.\right. \\
& \left.+\frac{K R+F_{0}}{\Gamma\left(\alpha_{1}+\beta_{1}\right)} \int_{0}^{\xi}(\xi-s)^{\alpha_{1}+\beta_{1}-1} \mathrm{~d} s+\frac{|\lambda|\left\|\psi_{1}\right\|_{E}}{\Gamma\left(\beta_{1}\right)} \int_{0}^{\xi}(\xi-s)^{\beta_{1}-1} \mathrm{~d} s\right) \\
& +\left|A_{2}\right|\left(\frac{|b|\left(K R+F_{0}\right)}{\Gamma\left(\bar{\alpha}_{1}\right)} \int_{0}^{\mu_{2}}\left(\mu_{2}-s\right)^{\bar{\alpha}_{1}-1} \mathrm{~d} s+\frac{|b \lambda|\left\|\psi_{1}\right\|_{E}}{\Gamma\left(\beta_{1}+q\right)} \int_{0}^{\mu_{2}}\left(\mu_{2}-s\right)^{\beta_{1}+q-1} \mathrm{~d} s\right. \\
& \left.\left.+\frac{L R+G_{0}}{\Gamma\left(\alpha_{2}+\beta_{2}\right)} \int_{0}^{\eta}(\eta-s)^{\alpha_{2}+\beta_{2}-1} \mathrm{~d} s+\frac{|k|\left\|\psi_{2}\right\|_{E}}{\Gamma\left(\beta_{2}\right)} \int_{0}^{\eta}(\eta-s)^{\beta_{2}-1} \mathrm{~d} s\right)+\left|A_{3}\right|\right\}+\left|a_{0}\right| \\
& \leq \frac{K R+F_{0}}{\Gamma\left(\alpha_{1}+\beta_{1}+1\right)}+\frac{\left|a A_{1}\right|\left(L R+G_{0}\right) \mu_{1}^{\bar{\alpha}_{2}}}{\Gamma\left(\bar{\alpha}_{2}+1\right)}+\frac{\left|A_{1}\right|\left(K R+F_{0}\right) \xi^{\alpha_{1}+\beta_{1}}}{\Gamma\left(\alpha_{1}+\beta_{1}+1\right)}+\frac{\left|b A_{2}\right|\left(K R+F_{0}\right) \mu_{2}^{\bar{\alpha}_{1}}}{\Gamma\left(\bar{\alpha}_{1}+1\right)} \\
& +\frac{\left|A_{2}\right|\left(L R+G_{0}\right) \eta^{\alpha_{2}-\beta_{2}}}{\Gamma\left(\alpha_{2}-\beta_{2}+1\right)}+\left|A_{3}\right|+\left|a_{0}\right|+\left(\frac{|\lambda|\left(1+\left|A_{1}\right| \xi^{\beta_{1}}\right)}{\Gamma\left(\beta_{1}+1\right)}+\frac{\left|b \lambda A_{2}\right| \mu_{2}^{\beta_{1}+q}}{\Gamma\left(\beta_{1}+q+1\right)}\right)\left\|\psi_{1}\right\|_{E} \\
& +\left(\frac{\left|k A_{2}\right| \eta^{\beta_{2}}}{\Gamma\left(\beta_{2}+1\right)}+\frac{\left|a k A_{1}\right| \mu_{1}^{\beta_{2}-p}}{\Gamma\left(\beta_{2}-p+1\right)}\right)\left\|\psi_{2}\right\|_{E} \\
& \leq\left(\frac{K\left(1+\left|A_{1}\right| \xi^{\alpha_{1}+\beta_{1}}\right)}{\Gamma\left(\alpha_{1}+\beta_{1}+1\right)}+\frac{\left|a A_{1}\right| \mu_{1}^{\bar{\alpha}_{2}} L}{\Gamma\left(\bar{\alpha}_{2}+1\right)}+\frac{\left|b A_{2}\right| \mu_{2}^{\bar{\alpha}_{1}} K}{\Gamma\left(\bar{\alpha}_{1}+1\right)}+\frac{\left|A_{2}\right| \eta^{\alpha_{2}+\beta_{2}} L}{\Gamma\left(\alpha_{2}+\beta_{2}+1\right)}\right) R \\
& +\frac{F_{0}\left(1+\left|A_{1}\right| \xi^{\alpha_{1}+\beta_{1}}\right)}{\Gamma\left(\alpha_{1}+\beta_{1}+1\right)}+\frac{\left|a A_{1}\right| \mu_{1}^{\bar{\alpha}_{2}} G_{0}}{\Gamma\left(\bar{\alpha}_{2}+1\right)}+\frac{\left|b A_{2}\right| \mu_{2}^{\bar{\alpha}_{1}} F_{0}}{\Gamma\left(\bar{\alpha}_{1}+1\right)}+\frac{\left|A_{2}\right| \eta^{\alpha_{2}+\beta_{2}} G_{0}}{\Gamma\left(\alpha_{2}+\beta_{2}+1\right)}+\left|A_{3}\right|+\left|a_{0}\right|+\rho_{1}\left\|\left(\psi_{1}, \psi_{2}\right)\right\|_{E \times E} \\
& \leq Q_{1} F_{0}+Q_{2} G_{0}+\left|A_{3}\right|+\left|a_{0}\right|+\left(Q_{1} K+Q_{2} L+\rho_{1}\right) R \text {. }
\end{aligned}
$$

On the other hand, we have 


$$
\begin{aligned}
\left|\mathfrak{R}_{2}\left(\psi_{1}, \psi_{2}\right)(t)\right| \leq & \frac{L R+G_{0}}{\Gamma\left(\alpha_{2}+\beta_{2}+1\right)}+\frac{\left|b B_{1}\right| \mu_{2}^{\bar{\alpha}_{1}}\left(K R+F_{0}\right)}{\Gamma\left(\bar{\alpha}_{1}+1\right)}+\frac{\left|B_{1}\right|\left(L R+G_{0}\right) \eta^{\alpha_{2}+\beta_{2}}}{\Gamma\left(\alpha_{2}+\beta_{2}+1\right)} \\
& +\frac{\left|a B_{2}\right|\left(L R+G_{0}\right) \mu_{1}^{\bar{\alpha}_{2}}}{\Gamma\left(\bar{\alpha}_{2}+1\right)}+\frac{\left|B_{2}\right|\left(K R+F_{0}\right) \xi^{\alpha_{1}+\beta_{1}}}{\Gamma\left(\alpha_{1}+\beta_{1}+1\right)}+\left|B_{3}\right|+\left|b_{0}\right|+\left(\frac{\left|b \lambda B_{1}\right| \mu_{2}^{\beta_{1}+q}}{\Gamma\left(\beta_{1}+q+1\right)}+\frac{\left|\lambda B_{2}\right| \xi^{\beta_{1}}}{\Gamma\left(\beta_{1}+1\right)}\right)\left\|\psi_{1}\right\|_{E} \\
& +\left(\frac{|k|}{\Gamma\left(\beta_{2}+1\right)}+\frac{\left|k B_{1}\right| \eta^{\beta_{2}}}{\Gamma\left(\beta_{2}+1\right)}+\frac{\left|a k B_{2}\right| \mu_{1}^{\beta_{2}-p}}{\Gamma\left(\beta_{2}-p+1\right)}\right)\left\|\psi_{2}\right\|_{E} \\
\leq & Q_{4} G_{0}+Q_{3} F_{0}+\left|B_{3}\right|+\left|b_{0}\right|+\left(Q_{4} L+Q_{3} K\right) R+\rho_{2}\left(\left\|\psi_{1}\right\|_{E}+\left\|\psi_{2}\right\|_{E}\right) \\
\leq & Q_{4} G_{0}+Q_{3} F_{0}+\left|B_{3}\right|+\left|b_{0}\right|+\left(Q_{4} L+Q_{3} K+\rho_{2}\right) R .
\end{aligned}
$$

Consequently,

$$
\begin{aligned}
\left\|\mathfrak{N}\left(\psi_{1}, \psi_{2}\right)\right\| & \leq\left(Q_{1}+Q_{3}\right) F_{0}+\left(Q_{2}+Q_{4}\right) G_{0}+\left|A_{3}\right|+\left|B_{3}\right|+\left|a_{0}\right|+\left|b_{0}\right|+\left[\left(Q_{1}+Q_{3}\right) K+\left(Q_{2}+Q_{4}\right) L+\rho_{1}+\rho_{2}\right] R \\
& \leq R .
\end{aligned}
$$

Therefore, $\mathfrak{N}\left(B_{R}\right) \subseteq B_{R}$. Next, we prove that $\mathfrak{N}$ is a contraction. Let $\left(\psi_{1}, \psi_{2}\right),\left(\bar{\psi}_{1}, \bar{\psi}_{2}\right) \in E \times E$. For all $t \in J$, we have

$$
\begin{aligned}
& \left|\mathfrak{N}_{1}\left(\psi_{1}, \psi_{2}\right)-\mathfrak{N}_{1}\left(\bar{\psi}_{1}, \bar{\psi}_{2}\right)(t)\right| \leq \frac{K\left(\left\|\psi_{1}-\bar{\psi}_{1}\right\|_{E}+\left\|\psi_{2}-\bar{\psi}_{2}\right\|_{E}\right)}{\Gamma\left(\alpha_{1}+\beta_{1}+1\right)} t^{\alpha_{1}+\beta_{1}}+\frac{|\lambda|\left\|\psi_{1}-\bar{\psi}_{1}\right\|_{E} \beta_{1}}{\Gamma\left(\beta_{1}+1\right)} \\
& +t^{\beta_{1}}\left\{| A _ { 1 } | \left(\frac{a L\left(\left\|\psi_{1}-\bar{\psi}_{1}\right\|_{E}+\left\|\psi_{2}-\bar{\psi}_{2}\right\|_{E}\right)}{\Gamma\left(\bar{\alpha}_{2}+1\right)} \mu_{1}^{\bar{\alpha}_{2}}+\frac{|a k|\left\|\psi_{2}-\bar{\psi}_{2}\right\|_{E}}{\Gamma\left(\beta_{2}-p+1\right)} \mu_{1}^{\beta_{2}-p}\right.\right. \\
& \left.+\frac{K\left(\left\|\psi_{1}-\bar{\psi}_{1}\right\|_{E}+\left\|\psi_{2}-\bar{\psi}_{2}\right\|_{E}\right)}{\Gamma\left(\alpha_{1}+\beta_{1}+1\right)} \xi^{\alpha_{1}+\beta_{1}}+\frac{|\lambda|\|x-\bar{x}\|_{E}}{\Gamma\left(\beta_{1}+1\right)} \xi^{\beta_{1}}\right) \\
& +\left|A_{2}\right|\left(\frac{|b| K\left(\left\|\psi_{1}-\bar{\psi}_{1}\right\|_{E}+\left\|\psi_{2}-\bar{\psi}_{2}\right\|_{E}\right)}{\Gamma\left(\bar{\alpha}_{1}+1\right)} \mu_{2}^{\bar{\alpha}_{1}}+\frac{|b \lambda|\left\|\psi_{1}-\bar{\psi}_{1}\right\|_{E}}{\Gamma\left(\beta_{1}+q+1\right)} \mu_{2}^{\beta_{1}+q}\right. \\
& \left.\left.+\frac{L\left(\left\|\psi_{1}-\bar{\psi}_{1}\right\|_{E}+\left\|\psi_{2}-\bar{\psi}_{2}\right\|_{E}\right)}{\Gamma\left(\alpha_{2}+\beta_{2}+1\right)} \eta^{\alpha_{2}+\beta_{2}}+\frac{|k|\left\|\psi_{2}-\bar{\psi}_{2}\right\|_{E}}{\Gamma\left(\beta_{2}+1\right)} \eta^{\beta_{2}}\right)+\left|A_{3}\right|\right\} \\
& \leq \frac{K\left\|\left(\psi_{1}-\bar{\psi}_{1}, \psi_{2}-\bar{\psi}_{2}\right)\right\|_{E \times E}}{\Gamma\left(\alpha_{1}+\beta_{1}+1\right)}+\frac{|\lambda|\left\|\psi_{1}-\bar{\psi}_{1}\right\|_{E}}{\Gamma\left(\beta_{1}+1\right)} \\
& +\frac{\left|a A_{1}\right| L\left\|\left(\psi_{1}-\bar{\psi}_{1}, \psi_{2}-\bar{\psi}_{2}\right)\right\|_{E \times E}}{\Gamma\left(\bar{\alpha}_{2}+1\right)} \mu_{1}^{\bar{\alpha}_{2}}+\frac{\left|a k A_{1}\right|\left\|\psi_{2}-\bar{\psi}_{2}\right\|_{E}}{\Gamma\left(\beta_{2}-p+1\right)} \mu_{1}^{\beta_{2}-p} \\
& +\frac{\left|A_{1}\right| K\left\|\left(\psi_{1}-\bar{\psi}_{1}, \psi_{2}-\bar{\psi}_{2}\right)\right\|_{E \times E}}{\Gamma\left(\alpha_{1}+\beta_{1}+1\right)} \xi^{\alpha_{1}+\beta_{1}}+\frac{\left|A_{1} \lambda\right|\left\|\psi_{1}-\bar{\psi}_{1}\right\|_{E}}{\Gamma\left(\beta_{1}+1\right)} \xi^{\beta_{1}} \\
& +\frac{\left|A_{2} b\right| K\left\|\left(\psi_{1}-\bar{\psi}_{1}, \psi_{2}-\bar{\psi}_{2}\right)\right\|_{E \times E}}{\Gamma\left(\bar{\alpha}_{1}+1\right)} \mu_{2}^{\bar{\alpha}_{1}}+\frac{\left|A_{2} b \lambda\right|\left\|\psi_{1}-\bar{\psi}_{1}\right\|_{E}}{\Gamma\left(\beta_{1}+q+1\right)} \mu_{2}^{\beta_{1}+q} \\
& +\frac{\left|A_{2}\right| L\left\|\left(\psi_{1}-\bar{\psi}_{1}, \psi_{2}-\bar{\psi}_{2}\right)\right\|_{E \times E}}{\Gamma\left(\alpha_{2}+\beta_{2}+1\right)} \eta^{\alpha_{2}+\beta_{2}}+\frac{\left|A_{2} k\right|\left\|\psi_{2}-\bar{\psi}_{2}\right\|_{E}}{\Gamma\left(\beta_{2}+1\right)} \eta^{\beta_{2}} \\
& \leq\left(Q_{1} K+Q_{2} L+\rho_{1}\right)\left\|\left(\psi_{1}, \psi_{2}\right)-\left(\bar{\psi}_{1}, \bar{\psi}_{2}\right)\right\|_{E \times E^{*}}
\end{aligned}
$$


In the other respect, we have

$$
\begin{aligned}
& \left|\mathfrak{N}_{2}\left(\psi_{1}, \psi_{2}\right)(t)-\mathfrak{N}_{2}\left(\bar{\psi}_{1}, \bar{\psi}_{2}\right)(t)\right| \leq \frac{L\left(\left\|\psi_{1}-\bar{\psi}_{1}\right\|_{E}+\left\|\psi_{2}-\bar{\psi}_{2}\right\|_{E}\right)}{\Gamma\left(\alpha_{2}+\beta_{2}+1\right)}+\frac{k\left\|\psi_{2}-\bar{\psi}_{2}\right\|_{E}}{\Gamma\left(\beta_{2}+1\right)} \\
& +\frac{\left|B_{1} b\right| K\left(\left\|\psi_{1}-\bar{\psi}_{1}\right\|_{E}+\left\|\psi_{2}-\bar{\psi}_{2}\right\|_{E}\right)}{\Gamma\left(\bar{\alpha}_{1}+1\right)} \mu_{2}^{\bar{\alpha}_{1}-1}+\frac{\left|B_{1} b \lambda\right|\left\|\psi_{1}-\bar{\psi}_{1}\right\|_{E}}{\Gamma\left(\beta_{1}+q+1\right)} \mu_{2}^{\beta_{1}+q} \\
& +\frac{\left|B_{1}\right| L\left(\left\|\psi_{1}-\bar{\psi}_{1}\right\|_{E}+\left\|\psi_{2}-\bar{\psi}_{2}\right\|_{E}\right)}{\Gamma\left(\alpha_{2}+\beta_{2}+1\right)} \eta^{\alpha_{2}+\beta_{2}}+\frac{\left|B_{1} k\right| K\left\|\psi_{2}-\bar{\psi}_{2}\right\|_{E}}{\Gamma\left(\beta_{2}+1\right)} \eta^{\beta_{2}} \\
& +\frac{\left|B_{2} a\right| L\left(\left\|\psi_{1}-\bar{\psi}_{1}\right\|_{E}+\left\|\psi_{2}-\bar{\psi}_{2}\right\|_{E}\right)}{\Gamma\left(\bar{\alpha}_{2}+1\right)} \mu_{1}^{\bar{\alpha}_{2}}+\frac{\left|B_{2} a k\right|\left\|\psi_{2}-\bar{\psi}_{2}\right\|_{E}}{\Gamma\left(\beta_{2}-p+1\right)} \mu_{1}^{\beta_{2}-p} \\
& +\frac{\left|B_{2}\right| K\left(\left\|\psi_{1}-\bar{\psi}_{1}\right\|_{E}+\left\|\psi_{2}-\bar{\psi}_{2}\right\|_{E}\right)}{\Gamma\left(\alpha_{1}+\beta_{1}+1\right)} \xi^{\alpha_{1}+\beta_{1}}+\frac{\left|B_{2} \lambda\right|\left\|\psi_{1}-\bar{\psi}_{1}\right\|_{E}}{\Gamma\left(\beta_{1}+1\right)} \xi^{\beta_{1}}, \\
& \leq\left(\frac{L}{\Gamma\left(\alpha_{2}+\beta_{2}+1\right)}+\frac{\left|B_{1} b\right| K}{\Gamma\left(\bar{\alpha}_{1}+1\right)} \mu_{2}^{\bar{\alpha}_{1}-1}+\frac{\left|B_{1}\right| L}{\Gamma\left(\alpha_{2}+\beta_{2}+1\right)} \eta^{\alpha_{2}+\beta_{2}}\right. \\
& \left.+\frac{\left|B_{2} a\right| L \mu_{1}^{\bar{\alpha}_{2}}}{\Gamma\left(\bar{\alpha}_{2}+1\right)}+\frac{\left|B_{2}\right| K \xi^{\alpha_{1}+\beta_{1}}}{\Gamma\left(\alpha_{1}+\beta_{1}+1\right)}\right)\left\|\left(\psi_{1}-\bar{\psi}_{1}, \psi_{2}-\bar{\psi}_{2}\right)\right\|_{E \times E} \\
& \left(\frac{\left|B_{1} b \lambda\right| \mu_{2}^{\beta_{1}+q}}{\Gamma\left(\beta_{1}+q+1\right)}+\frac{\left|B_{2} \lambda\right| \xi^{\beta_{1}}}{\Gamma\left(\beta_{1}+1\right)}\right)\left\|\psi_{1}-\bar{\psi}_{1}\right\|_{E} \\
& \left(\frac{|k|\left(1+\left|B_{1}\right| K \eta^{\beta_{2}}\right)}{\Gamma\left(\beta_{2}+1\right)}+\frac{\left|B_{2} a k\right| \mu_{1}^{\beta_{2}-p}}{\Gamma\left(\beta_{2}-p+1\right)}\right)\left\|\psi_{2}-\bar{\psi}_{2}\right\|_{E} \\
& \leq\left(Q_{4} L+Q_{3} K+\rho_{2}\right)\left\|\left(\psi_{1}, \psi_{2}\right)-\left(\bar{\psi}_{1}, \bar{\psi}_{2}\right)\right\|_{E \times E^{*}}
\end{aligned}
$$

Consequently,

$$
\begin{aligned}
\left\|\mathfrak{N}\left(\psi_{1}, \psi_{2}\right)-\mathfrak{N}(\bar{x}, \bar{y})\right\| \leq & {\left[\left(Q_{1}+Q_{3}\right) K+\left(Q_{2}+Q_{4}\right) L\right.} \\
& \left.+\rho_{1}+\rho_{2}\right]\left\|\left(\psi_{1}, \psi_{2}\right)-\left(\bar{\psi}_{1}, \bar{\psi}_{2}\right)\right\|_{E \times E^{*}}
\end{aligned}
$$

Therefore, the operator $\mathfrak{N}$ has a unique fixed point. Thus, we conclude that problems (1)-(2) have a unique solution on $[0,1]$. The proof is complete.

Now, we apply the Leray-Schauder alternative theoerm to obtain the following result about the existence of solutions for problems (1)-(2).

Theorem 10. Assume that (H1) holds. If

$$
S<1 \text {, and } \bar{S}<1 \text {, }
$$

where

$$
\begin{aligned}
& S=Q_{1} \sigma_{1}+Q_{2} \tau_{1}+Q_{3} \sigma_{1}+Q_{4} \tau_{1}+Q_{5}+Q_{8}, \\
& \bar{S}=Q_{1} \sigma_{2}+Q_{2} \tau_{2}+Q_{3} \sigma_{2}+Q_{4} \tau_{2}+Q_{6}+Q_{7},
\end{aligned}
$$

then the boundary value problems (1)-(2) have at least one solution on $[0,1]$.

Proof. First, we show that the operator $\mathfrak{N}$ is completely continuous.

Because $f$ and $g$ are continuous functions, $\mathfrak{N}$ is continuous operator as well. Let $\Lambda$ be any nonempty bounded subset of $E \times E$. Then, there exists $r>0$ such that for any $\left(\psi_{1}, \psi_{2}\right) \in \Lambda,\left\|\left(\psi_{1}, \psi_{2}\right)\right\|_{E \times E} \leq r$. Notice that from the condition (H1) for all $\left(\psi_{1}, \psi_{2}\right) \in \Lambda$, we have

$$
\begin{aligned}
\left|f\left(t, \psi_{1}(t), \psi_{2}(t)\right)\right| & \leq \sigma_{0}+\sigma_{1}\left|\psi_{1}(t)\right|+\sigma_{2}\left|\psi_{2}(t)\right|, \\
& \leq \sigma_{0}+\max \left\{\sigma_{1}, \sigma_{2}\right\}\left\|\left(\psi_{1}, \psi_{2}\right)\right\|_{E \times E} \\
& \leq \sigma_{0}+r \max \left\{\sigma_{1}, \sigma_{2}\right\} \\
\left|g\left(t, \psi_{1}(t), \psi_{2}(t)\right)\right| & \leq \tau_{0}+r \max \left\{\tau_{1}, \tau_{2}\right\} .
\end{aligned}
$$

Next, we prove that $\mathfrak{N}(\Lambda)$ is uniformly bounded. Let $\left(\psi_{1}, \psi_{2}\right) \in \Lambda$. Indeed, for any $t \in[0,1]$, we have 


$$
\begin{aligned}
\left|\Re_{1}\left(\psi_{1}, \psi_{2}\right)(t)\right| \leq & \frac{\sigma_{0}+r \max \left\{\sigma_{1}, \sigma_{2}\right\}}{\Gamma\left(\alpha_{1}+\beta_{1}\right)} \int_{0}^{t}(t-s)^{\alpha_{1}+\beta_{1}-1} \mathrm{~d} s+\frac{|\lambda|\left\|\psi_{1}\right\|_{E}}{\Gamma\left(\beta_{1}\right)} \int_{0}^{t}(t-s)^{\beta_{1}-1} \mathrm{~d} s \\
& +t^{\beta_{1}}\left\{| A _ { 1 } | \left(\frac{|a|\left(\tau_{0}+r \max \left\{\tau_{1}, \tau_{2}\right\}\right)}{\Gamma\left(\bar{\alpha}_{2}\right)} \int_{0}^{\mu_{1}}\left(\mu_{1}-s\right)^{\bar{\alpha}_{2}-1} \mathrm{~d} s+\frac{|a k|\left\|\psi_{2}\right\|_{E}}{\Gamma\left(\beta_{2}-p\right)} \int_{0}^{\mu_{1}}\left(\mu_{1}-s\right)^{\beta_{2}-p-1} \mathrm{~d} s\right.\right. \\
& \left.+\frac{\sigma_{0}+r \max \left\{\sigma_{1}, \sigma_{2}\right\}}{\Gamma\left(\alpha_{1}+\beta_{1}\right)} \int_{0}^{\xi}(\xi-s)^{\alpha_{1}+\beta_{1}-1} \mathrm{~d} s+\frac{|\lambda|\left\|\psi_{1}\right\|_{E}}{\Gamma\left(\beta_{1}\right)} \int_{0}^{\xi}(\xi-s)^{\beta_{1}-1} \mathrm{~d} s\right) \\
& +\left|A_{2}\right|\left(\frac{|b|\left(\sigma_{0}+r \max \left\{\sigma_{1}, \sigma_{2}\right\}\right)}{\Gamma\left(\bar{\alpha}_{1}\right)} \int_{0}^{\mu_{2}}\left(\mu_{2}-s\right)^{\bar{\alpha}_{1}-1} \mathrm{~d} s+\frac{|b \lambda||| \psi_{1} \|_{E}}{\Gamma\left(\beta_{1}+q\right)} \int_{0}^{\mu_{2}}\left(\mu_{2}-s\right)^{\beta_{1}+q-1} \mathrm{~d} s\right. \\
& \left.\left.+\frac{\tau_{0}+r \max \left\{\tau_{1}, \tau_{2}\right\}}{\Gamma\left(\alpha_{2}+\beta_{2}\right)} \int_{0}^{\eta}(\eta-s)^{\alpha_{2}+\beta_{2}-1} \mathrm{~d} s+\frac{|k| \mid \psi_{2} \|_{E}}{\Gamma\left(\beta_{2}\right)} \int_{0}^{\eta}(\eta-s)^{\beta_{2}-1} \mathrm{~d} s\right)+\left|A_{3}\right|\right\}+\left|a_{0}\right|, \\
\leq & \frac{\left(1+\left|A_{1}\right| \xi_{1}^{\alpha_{1}+\beta_{1}}\right)\left(\sigma_{0}+r \max \left\{\sigma_{1}, \sigma_{2}\right\}\right)}{\Gamma\left(\alpha_{1}+\beta_{1}+1\right)}+\frac{\left|a A_{1}\right|\left(\tau_{0}+r \max \left\{\tau_{1}, \tau_{2}\right\}\right) \mu_{1}^{\bar{\alpha}_{2}}}{\Gamma\left(\bar{\alpha}_{2}+1\right)} \\
& +\frac{\left|b A_{2}\right|\left(\sigma_{0}+r \max \left\{\sigma_{1}, \sigma_{2}\right\}\right) \mu_{2}^{\bar{\alpha}_{1}}}{\Gamma\left(\bar{\alpha}_{1}+1\right)}+\frac{\left|A_{2}\right|\left(\tau_{0}+r \max \left\{\tau_{1}, \tau_{2}\right\}\right) \eta^{\alpha_{2}+\beta_{2}}}{\Gamma\left(\alpha_{2}+\beta_{2}+1\right)}+\left|A_{3}\right|+\left|a_{0}\right| \\
& +\left(\frac{|\lambda|\left(1+\left|A_{1}\right| \xi^{\beta_{1}}\right)}{\Gamma\left(\beta_{1}+1\right)}+\frac{\left|b \lambda A_{2}\right| \mu_{2}^{\beta_{1}+q}}{\Gamma\left(\beta_{1}+q+1\right)}\right)\left\|\psi_{1}\right\|_{E}+\left(\frac{\left|k A_{2}\right| \eta^{\beta_{2}}}{\Gamma\left(\beta_{2}+1\right)}+\frac{\left|a k A_{1}\right| \mu_{1}^{\beta_{2}-p}}{\Gamma\left(\beta_{2}-p+1\right)}\right)\left\|\psi_{2}\right\|_{E} \\
\leq & \left.+\infty \frac{\left(1+\left|A_{1}\right| \xi^{\alpha_{1}+\beta_{1}}\right)}{\Gamma\left(\alpha_{1}+\beta_{1}+1\right)}+\frac{\left|b A_{2}\right| \mu_{2}^{\bar{\alpha}_{1}}}{\Gamma\left(\bar{\alpha}_{1}+1\right)}\right)\left(\sigma_{0}+r \max \left\{\sigma_{1}, \sigma_{2}\right\}\right) \\
& +\left(\frac{\left|a A_{1}\right| \bar{\mu}_{1}}{\Gamma\left(\bar{\alpha}_{2}+1\right)}+\frac{\left|A_{2}\right| \eta^{\alpha_{2}+\beta_{2}}}{\Gamma\left(\alpha_{2}+\beta_{2}+1\right)}\right)\left(\tau_{0}+r \max \left\{\tau_{1}, \tau_{2}\right\}\right)+\left|A_{3}\right|+\left|a_{0}\right|+\rho_{1} r \\
\leq &
\end{aligned}
$$

Similarly,

$$
\begin{aligned}
\left|\mathfrak{N}_{2}\left(\psi_{1}, \psi_{2}\right)(t)\right|= & \frac{\left(1+\left|B_{1}\right| \eta^{\alpha_{2}+\beta_{2}}\right)\left(\tau_{0}+r \max \left\{\tau_{1}, \tau_{2}\right\}\right)}{\Gamma\left(\alpha_{2}+\beta_{2}+1\right)}+\frac{\left|b B_{1}\right| \mu_{2}^{\bar{\alpha}_{1}}\left(\sigma_{0}+r \max \left\{\sigma_{1}, \sigma_{2}\right\}\right)}{\Gamma\left(\bar{\alpha}_{1}+1\right)} \\
& +\frac{\left|a B_{2}\right|\left(\tau_{0}+r \max \left\{\tau_{1}, \tau_{2}\right\}\right) \mu_{1}^{\bar{\alpha}_{2}}}{\Gamma\left(\bar{\alpha}_{2}+1\right)}+\frac{\left|B_{2}\right|\left(\sigma_{0}+r \max \left\{\sigma_{1}, \sigma_{2}\right\}\right) \xi^{\alpha_{1}+\beta_{1}}}{\Gamma\left(\alpha_{1}+\beta_{1}+1\right)}+\left|B_{3}\right|+\left|b_{0}\right| \\
& +\left(\frac{\left|b \lambda B_{1}\right| \mu_{2}^{\beta_{1}+q}}{\Gamma\left(\beta_{1}+q+1\right)}+\frac{\left|\lambda B_{2}\right| \xi^{\beta_{1}}}{\Gamma\left(\beta_{1}+1\right)}\right)\left\|\psi_{1}\right\|_{E}+\left(\frac{|k|+\left|k B_{1}\right| \eta^{\beta_{2}}}{\Gamma\left(\beta_{2}+1\right)}+\frac{\left|a k B_{2}\right| \mu_{1}^{\beta_{2}-p}}{\Gamma\left(\beta_{2}-p+1\right)}\right)\left\|\psi_{2}\right\|_{E} \\
\leq & \left(\frac{\left|b B_{1}\right| \mu_{2}^{\bar{\alpha}_{1}}}{\Gamma\left(\bar{\alpha}_{1}+1\right)}+\frac{\left|B_{2}\right| \xi^{\alpha_{1}+\beta_{1}}}{\Gamma\left(\alpha_{1}+\beta_{1}+1\right)}\right)\left(\sigma_{0}+r \max \left\{\sigma_{1}, \sigma_{2}\right\}\right) \\
& +\left(\frac{\left|a B_{2}\right| \mu_{1}^{\bar{\alpha}_{2}}}{\Gamma\left(\bar{\alpha}_{2}+1\right)}+\frac{\left(1+\left|B_{1}\right| \eta^{\alpha_{2}+\beta_{2}}\right)}{\Gamma\left(\alpha_{2}+\beta_{2}+1\right)}\right)\left(\tau_{0}+r \max \left\{\tau_{1}, \tau_{2}\right\}\right)+\left|B_{3}\right|+\left|b_{0}\right|+\rho_{2} r \\
& <+\infty .
\end{aligned}
$$


Consequently, $\left\|\left(\psi_{1}, \psi_{2}\right)\right\|_{E \times E}<+\infty$ for any $\left(\psi_{1}, \psi_{2}\right) \in \Lambda$. Therefore, $\mathfrak{N}(\Lambda)$ is uniformly bounded.
Now, we show that $\mathfrak{N}$ is equicontinuous on $\Lambda$. Let $\left(\psi_{1}, \psi_{2}\right) \in \Lambda$. For any $t_{2}, t_{1} \in J$, where $t_{2}>t_{1}$, we have

$$
\begin{aligned}
& \left|\mathfrak{N}_{1}\left(\psi_{1}, \psi_{2}\right)\left(t_{2}\right)-\mathfrak{N}_{1}\left(\psi_{1}, \psi_{2}\right)\left(t_{1}\right)\right| \\
& \leq \frac{\sigma_{0}+r \max \left\{\sigma_{1}, \sigma_{2}\right\}}{\Gamma\left(\alpha_{1}+\beta_{1}\right)}\left(\int_{0}^{t_{1}}\left[\left(t_{2}-s\right)^{\alpha_{1}+\beta_{1}-1}-\left(t_{1}-s\right)^{\alpha_{1}+\beta_{1}-1}\right] \mathrm{d} s+\int_{t_{1}}^{t_{2}}\left(t_{2}-s\right)^{\alpha_{1}+\beta_{1}-1} \mathrm{~d} s\right) \\
& +\frac{|\lambda|\left\|\psi_{1}\right\|_{E}}{\Gamma\left(\beta_{1}\right)}\left(\int_{0}^{t_{1}}\left[\left(t_{2}-s\right)^{\beta_{1}-1}-\left(t_{1}-s\right)^{\beta_{1}-1}\right] \mathrm{d} s+\int_{t_{1}}^{t_{2}}\left(t_{2}-s\right)^{\beta_{1}-1} \mathrm{~d} s\right) \\
& +\left(t_{2}^{\beta_{1}}-t_{1}^{\beta_{1}}\right)\left\{| A _ { 1 } | \left(\frac{|a|\left(\tau_{0}+r \max \left\{\tau_{1}, \tau_{2}\right\}\right)}{\Gamma\left(\bar{\alpha}_{2}\right)} \int_{0}^{\mu_{1}}\left(\mu_{1}-s\right)^{\bar{\alpha}_{2}-1} \mathrm{~d} s+\frac{|a k|\left\|\psi_{2}\right\|_{E}}{\Gamma\left(\beta_{2}-p\right)} \int_{0}^{\mu_{1}}\left(\mu_{1}-s\right)^{\beta_{2}-p-1} \mathrm{~d} s\right.\right. \\
& \left.+\frac{\sigma_{0}+r \max \left\{\sigma_{1}, \sigma_{2}\right\}}{\Gamma\left(\alpha_{1}+\beta_{1}\right)} \int_{0}^{\xi}(\xi-s)^{\alpha_{1}+\beta_{1}-1} \mathrm{~d} s+\frac{|\lambda|\left\|\psi_{1}\right\|_{E}}{\Gamma\left(\beta_{1}\right)} \int_{0}^{\xi}(\xi-s)^{\beta_{1}-1} \mathrm{~d} s\right) \\
& +\left|A_{2}\right|\left(\frac{|b|\left(\sigma_{0}+r \max \left\{\sigma_{1}, \sigma_{2}\right\}\right)}{\Gamma\left(\bar{\alpha}_{1}\right)} \int_{0}^{\mu_{2}}\left(\mu_{2}-s\right)^{\bar{\alpha}_{1}-1} \mathrm{~d} s+\frac{|b \lambda|\left\|\psi_{1}\right\|_{E}}{\Gamma\left(\beta_{1}+q\right)} \int_{0}^{\mu_{2}}\left(\mu_{2}-s\right)^{\beta_{1}+q-1} \mathrm{~d} s\right. \\
& \left.\left.+\frac{\tau_{0}+r \max \left\{\tau_{1}, \tau_{2}\right\}}{\Gamma\left(\alpha_{2}+\beta_{2}\right)} \int_{0}^{\eta}(\eta-s)^{\alpha_{2}+\beta_{2}-1} \mathrm{~d} s+\frac{|k|\left\|\psi_{2}\right\|_{E}}{\Gamma\left(\beta_{2}\right)} \int_{0}^{\eta}(\eta-s)^{\beta_{2}-1} d s\right)+\left|A_{3}\right|\right\} \\
& \leq \frac{\left[\sigma_{0}+r \max \left\{\sigma_{1}, \sigma_{2}\right\}\right]}{\Gamma\left(\alpha_{1}+\beta_{1}+1\right)}\left(-\left(t_{2}-t_{1}\right)^{\alpha_{1}+\beta_{1}}+t_{2}^{\alpha_{1}+\beta_{1}}-t_{1}^{\alpha_{1}+\beta_{1}}+\left(t_{2}-t_{1}\right)^{\alpha_{1}+\beta_{1}}\right) \\
& +\frac{|\lambda|\left\|\psi_{1}\right\|_{E}}{\Gamma\left(\beta_{1}+1\right)}\left(-\left(t_{2}-t_{1}\right)^{\beta_{1}}+t_{2}^{\beta_{1}}-t_{1}^{\beta_{1}}+\left(t_{2}-t_{1}\right)^{\beta_{1}}\right)+\left(t_{2}^{\beta_{1}}-t_{1}^{\beta_{1}}\right) \\
& \left\{\left(\frac{\left|A_{1}\right||a| \mu_{1}^{\bar{\alpha}_{2}}}{\Gamma\left(\bar{\alpha}_{2}+1\right)}+\frac{\left|A_{2}\right| \eta^{\alpha_{2}+\beta_{2}}}{\Gamma\left(\alpha_{2}+\beta_{2}+1\right)}\right)\left(\tau_{0}+r \max \left\{\tau_{1}, \tau_{2}\right\}\right)\right. \\
& +\left(\frac{\left|A_{1}\right||a k| \mu_{1}^{\beta_{2}-p}}{\Gamma\left(\beta_{2}-p+1\right)}+\frac{\left|A_{2}\right||k| \eta^{\beta_{2}}}{\Gamma\left(\beta_{2}+1\right)}\right)\left\|\psi_{2}\right\|_{E}+\left(\frac{\left|A_{1}\right||\lambda| \xi^{\beta_{1}}}{\Gamma\left(\beta_{1}+1\right)}++\frac{\left|A_{2}\right||b \lambda| \mu_{2}^{\beta_{1}+q}}{\Gamma\left(\beta_{1}+q+1\right)}\right)\left\|\psi_{1}\right\|_{E} \\
& \left.+\left(\frac{\left|A_{2}\right||b| \mu_{2}^{\bar{\alpha}_{1}}}{\Gamma\left(\bar{\alpha}_{1}+1\right)}+\frac{\left|A_{1}\right| \xi^{\alpha_{1}+\beta_{1}}}{\Gamma\left(\alpha_{1}+\beta_{1}+1\right)}\right)\left(\sigma_{0}+r \max \left\{\sigma_{1}, \sigma_{2}\right\}\right)+\left|A_{3}\right|\right\} \\
& \leq \frac{\sigma_{0}+r \max \left\{\sigma_{1}, \sigma_{2}\right\}}{\Gamma\left(\alpha_{1}+\beta_{1}+1\right)}\left(t_{2}^{\alpha_{1}+\beta_{1}}-t_{1}^{\alpha_{1}+\beta_{1}}\right)+\frac{|\lambda| r}{\Gamma\left(\beta_{1}+1\right)}\left(t_{2}^{\beta_{1}}-t_{1}^{\beta_{1}}\right) \\
& +\left(t_{2}^{\beta_{1}}-t_{1}^{\beta_{1}}\right)\left\{\left(\frac{\left|A_{1}\right||a| \mu_{1}^{\bar{\alpha}_{2}}}{\Gamma\left(\bar{\alpha}_{2}+1\right)}+\frac{\left|A_{2}\right| \eta^{\alpha_{2}+\beta_{2}}}{\Gamma\left(\alpha_{2}+\beta_{2}+1\right)}\right)\left(\tau_{0}+r \max \left\{\tau_{1}, \tau_{2}\right\}\right)\right. \\
& +r \max \left\{\left(\frac{\left|A_{1}\right||a k| \mu_{1}^{\beta_{2}-p}}{\Gamma\left(\beta_{2}-p+1\right)}+\frac{\left|A_{2}\right||k| \eta^{\beta_{2}}}{\Gamma\left(\beta_{2}+1\right)}\right),\left(\frac{\left|A_{1}\right||\lambda| \xi^{\beta_{1}}}{\Gamma\left(\beta_{1}+1\right)}+\frac{\left|A_{2}\right||b \lambda| \mu_{2}^{\beta_{1}+q}}{\Gamma\left(\beta_{1}+q+1\right)}\right)\right\} \\
& \left.+\left(\frac{\left|A_{2}\right||b| \mu_{2}^{\bar{\alpha}_{1}}}{\Gamma\left(\bar{\alpha}_{1}+1\right)}+\frac{\left|A_{1}\right| \xi^{\alpha_{1}+\beta_{1}}}{\Gamma\left(\alpha_{1}+\beta_{1}+1\right)}\right)\left(\sigma_{0}+r \max \left\{\sigma_{1}, \sigma_{2}\right\}\right)+\left|A_{3}\right|\right\} \\
& \longrightarrow 0, \quad \text { as } t_{2} \longrightarrow t_{1} \text {. }
\end{aligned}
$$

Analogously, 


$$
\begin{aligned}
\left|\mathfrak{N}_{2}\left(\psi_{1}, \psi_{2}\right)\left(t_{2}\right)-\mathfrak{N}_{2}\left(\psi_{1}, \psi_{2}\right)\left(t_{1}\right)\right| \leq & \frac{\tau_{0}+r \max \left\{\tau_{1}, \tau_{2}\right\}}{\Gamma\left(\alpha_{2}+\beta_{2}+1\right)}\left(t_{2}^{\alpha_{2}+\beta_{2}}-t_{1}^{\alpha_{2}+\beta_{2}}\right)+\frac{|k| r}{\Gamma\left(\beta_{2}+1\right)}\left(t_{2}^{\beta_{2}}-t_{1}^{\beta_{2}}\right) \\
& +\left(t_{2}^{\beta_{2}}-t_{1}^{\beta_{2}}\right)\left\{\left(\frac{\left|b B_{1}\right| \mu_{2}^{\bar{\alpha}_{1}}}{\Gamma\left(\bar{\alpha}_{1}+1\right)}+\frac{B_{2} \xi^{\alpha_{1}+\beta_{1}}}{\Gamma\left(\alpha_{1}+\beta_{1}+1\right)}\right)\left(\sigma_{0}+r \max \left\{\sigma_{1}, \sigma_{2}\right\}\right)\right. \\
& +r \max \left\{\left(\frac{\left|B_{2} \lambda\right| \xi^{\beta_{1}}}{\Gamma\left(\beta_{1}+1\right)}+\frac{\left|b \lambda B_{1}\right| \mu_{2}^{\beta_{1}+q}}{\Gamma\left(\beta_{1}+q+1\right)}\right),\left(\frac{\left|B_{2} a k\right| \mu_{1}^{\beta_{2}-p}}{\Gamma\left(\beta_{2}-p+1\right)}+\frac{\left|B_{1} k\right| \eta^{\beta_{2}}}{\Gamma\left(\beta_{2}+1\right)}\right)\right\} \\
& \left.+\left(\frac{\left|B_{1}\right| \eta^{\alpha_{2}+\beta_{2}}}{\Gamma\left(\alpha_{2}+\beta_{2}+1\right)}+\frac{\left|B_{2} a\right| \mu_{1}^{\bar{\alpha}_{2}}}{\Gamma\left(\bar{\alpha}_{2}+1\right)}\right)\left(\tau_{0}+r \max \left\{\tau_{1}, \tau_{2}\right\}\right)+\left|B_{3}\right|\right\} \\
& \longrightarrow 0, \quad \text { as } t_{2} \longrightarrow t_{1},
\end{aligned}
$$

which imply that $\left|\mathfrak{N}\left(\psi_{1}, \psi_{2}\right)\left(t_{2}\right)-\mathfrak{N}\left(\psi_{1}, \psi_{2}\right)\left(t_{1}\right)\right| \longrightarrow 0$ as $t_{2} \longrightarrow t_{1}$. Thus, the operator $\mathfrak{N}$ is equicontinuous. Hence, by Arzela-Ascoli theorem, we deduce that the operator $\mathfrak{N}$ is completely continuous.
Finally, we will verify that the set $M(\mathfrak{N})$ $=\left\{\left(\psi_{1}, \psi_{2}\right) \in E \times E:\left(\psi_{1}, \psi_{2}\right)=m \mathfrak{N}\left(\psi_{1}, \psi_{2}\right)\right.$ for some $0<m$ $<1\}$ is bounded. For all $\left(\psi_{1}, \psi_{2}\right) \in M(\mathfrak{N})$ and for any $t \in J$, we have $m \mathfrak{N}\left(\psi_{1}, \psi_{2}\right)=\left(m \mathfrak{N}_{1}\left(\psi_{1}, \psi_{2}\right), m \mathfrak{N}_{2}\left(\psi_{1}, \psi_{2}\right)\right)$. Then,

$$
\begin{aligned}
\left|\psi_{1}(t)\right|= & m\left|\mathfrak{N}_{1}\left(\psi_{1}, \psi_{2}\right)(t)\right| \leq \frac{\sigma_{0}+\sigma_{1}\left\|\psi_{1}\right\|_{E}+\sigma_{2}\left\|\psi_{2}\right\|_{E}}{\Gamma\left(\alpha_{1}+\beta_{1}+1\right)}+\left(\frac{\left|A_{1}\right||a| \mu_{1}^{\bar{\alpha}_{2}}}{\Gamma\left(\bar{\alpha}_{2}+1\right)}+\frac{\left|A_{2}\right| \eta^{\alpha_{2}+\beta_{2}}}{\Gamma\left(\alpha_{2}+\beta_{2}+1\right)}\right) \\
& \times\left(\tau_{0}+\tau_{1}\left\|\psi_{1}\right\|_{E}+\tau_{2}\left\|\psi_{2}\right\|_{E}\right)+\left(\frac{\left|A_{1}\right||a k| \mu_{1}^{\beta_{2}-p}}{\Gamma\left(\beta_{2}-p+1\right)}+\frac{\left|A_{2}\right||k| \eta^{\beta_{2}}}{\Gamma\left(\beta_{2}+1\right)}\right)\left\|\psi_{2}\right\|_{E} \\
& +\left(\frac{\left|A_{1}\right||\lambda| \xi^{\beta_{1}}}{\Gamma\left(\beta_{1}+1\right)}+\frac{\left|A_{2}\right||b \lambda| \mu_{2}^{\beta_{1}+q}}{\Gamma\left(\beta_{1}+q+1\right)}+\frac{|\lambda|}{\Gamma\left(\beta_{1}+1\right)}\right)\left\|\psi_{1}\right\|_{E} \\
& +\left(\frac{\left|A_{2}\right||b| \mu_{2}^{\bar{\alpha}_{1}}}{\Gamma\left(\bar{\alpha}_{1}+1\right)}+\frac{\left|A_{1}\right| \xi^{\alpha_{1}+\beta_{1}}}{\Gamma\left(\alpha_{1}+\beta_{1}+1\right)}\right)\left[\sigma_{0}+\sigma_{1}\left\|\psi_{1}\right\|_{E}+\sigma_{2}\left\|\psi_{2}\right\|_{E}\right]+\left|A_{3}\right|+\left|a_{0}\right| .
\end{aligned}
$$

This yields that

$$
\begin{aligned}
\left\|\psi_{1}\right\|_{E} & =m\left\|\mathfrak{N}_{1}\left(\psi_{1}, \psi_{2}\right)\right\| \\
& \leq Q_{1}\left(\sigma_{0}+\sigma_{1}\left\|\psi_{1}\right\|_{E}+\sigma_{2}\left\|\psi_{2}\right\|_{E}\right)+Q_{2}\left(\tau_{0}+\tau_{1}\left\|\psi_{1}\right\|_{E}+\tau_{2}\left\|\psi_{2}\right\|_{E}\right)+Q_{5}\left\|\psi_{1}\right\|_{E}+Q_{6}\left\|\psi_{2}\right\|_{E}+\left|A_{3}\right|+\left|a_{0}\right| .
\end{aligned}
$$

In the same way, we deduce that

$$
\begin{aligned}
\left\|\psi_{2}\right\|_{E} & =m\left\|\mathfrak{N}_{2}\left(\psi_{1}, \psi_{2}\right)\right\| \\
& \leq Q_{4}\left(\tau_{0}+\tau_{1}\left\|\psi_{1}\right\|_{E}+\tau_{2}\left\|\psi_{2}\right\|_{E}\right)+Q_{3}\left(\sigma_{0}+\sigma_{1}\left\|\psi_{1}\right\|_{E}+\sigma_{2}\left\|\psi_{2}\right\|_{E}\right)+Q_{7}\left\|\psi_{2}\right\|_{E}+Q_{8}\left\|\psi_{1}\right\|_{E}+\left|B_{3}\right|+\left|b_{0}\right| .
\end{aligned}
$$

Hence, we have 


$$
\begin{aligned}
\left\|\left(\psi_{1}, \psi_{2}\right)\right\|_{E \times E}= & \left\|\psi_{1}\right\|_{E}+\left\|\psi_{2}\right\|_{E} \\
\leq & Q_{1}\left[\sigma_{0}+\sigma_{1}\left\|\psi_{1}\right\|_{E}+\sigma_{2}\left\|\psi_{2}\right\|_{E}+Q_{2}\left(\tau_{0}+\tau_{1}\left\|\psi_{1}\right\|_{E}+\tau_{2}\left\|\psi_{2}\right\|_{E}\right)\right. \\
& +Q_{5}\left\|\psi_{1}\right\|_{E}+Q_{6}\left\|\psi_{2}\right\|_{E}+Q_{4}\left(\tau_{0}+\tau_{1}\left\|\psi_{1}\right\|_{E}+\tau_{2}\left\|\psi_{2}\right\|_{E}\right)+Q_{3}\left(\sigma_{0}+\sigma_{1}\left\|\psi_{1}\right\|_{E}+\sigma_{2}\left\|\psi_{2}\right\|_{E}\right) \\
& +Q_{7}\left\|\psi_{2}\right\|_{E}+Q_{8}\left\|\psi_{1}\right\|_{E}+\left|A_{3}\right|+\left|B_{3}\right|+\left|a_{0}\right|+\left|b_{0}\right|,
\end{aligned}
$$

which yields

$$
\begin{aligned}
& \left\|\left(\psi_{1}, \psi_{2}\right)\right\|_{E \times E} \\
& \leq \frac{Q_{3} \sigma_{0}+Q_{4} \tau_{0}+Q_{1} \sigma_{0}+Q_{2} \tau_{0}+\left|A_{3}\right|+\left|B_{3}\right|+\left|a_{0}\right|+\left|b_{0}\right|}{1-\max \{S, \bar{S}\}},
\end{aligned}
$$

where $S$ and $\bar{S}$ are given by (36) and (38), respectively, which proves that $M(\mathfrak{N})$ is bounded. Thus, as a consequence of Leray-Schauder alternative theorem, $\mathfrak{N}$ has more than one fixed point. Hence, the boundary value problems (1)-(2) have one solution at the very least on $[0,1]$.

\section{Applications and Numerical Examples}

In this section, we solve the integral equations (12) and (13) using the Adams-type predictor-corrector method with step size $h=0.01$ (for details, see [16-18] and the references therein). Briefly, we aim to approximate the solution of the following fractional initial value problem:

$$
D^{\alpha} y(t)=f(t, y(t)), y\left(t_{0}\right)=y_{0}, t \in\left(t_{0}, T\right], \quad \alpha \in(0,1),
$$

$$
y\left(t_{m}\right) \approx Y\left(t_{m}\right)=y\left(t_{0}\right)+\frac{1}{\Gamma(\alpha)} \sum_{j=0}^{m} R_{m, j} f_{j},
$$

where

$$
\begin{aligned}
R_{m, j} & = \begin{cases}W 0_{m, 0}, & \text { if } j=0, \\
W 1_{m, j-1}+W 0_{m, j}, & \text { if } 1 \leq j \leq m-1, \\
W 1_{m, m-1}, & \text { if } j=m .\end{cases} \\
W 1_{m, j} & =\frac{(-\alpha+j-m)(h(-j+m-1))^{\alpha}+(m-j)(h(m-j))^{\alpha}}{\alpha(\alpha+1)} \\
W 0_{m, j} & =\frac{(\alpha+j-m+1)(h(m-j))^{\alpha}+(h(-j+m-1))^{\alpha+1} / h}{\alpha(\alpha+1)} .
\end{aligned}
$$

To measure the accuracy of the present algorithm, we calculated the residual function as follows:

$$
R(t):=D^{\alpha} Y(t)-f(t, Y(t)),
$$

at the grid points $t_{m}$ for $m>0$, i.e.,

at the grid points $t_{m}=t_{0}+m h, m \geq 0$, with $h$ is a uniform step size. It is found that

$$
\begin{aligned}
R\left(t_{m}\right) & =\frac{1}{\Gamma(1-\alpha)} \int_{t_{0}}^{t_{m}}\left(t_{m}-\tau\right)^{-\alpha} Y^{\prime}(\tau) \mathrm{d} \tau-f\left(t_{m}, Y\left(t_{m}\right)\right), \\
& =\frac{1}{\Gamma(1-\alpha)} \sum_{j=0}^{m-1} \int_{t_{j}}^{t_{j+1}}\left(t_{m}-\tau\right)^{-\alpha} Y^{\prime}(\tau) \mathrm{d} \tau-f\left(t_{m}, Y\left(t_{m}\right)\right), \\
& =\frac{h^{-\alpha}}{2 \Gamma(1-\alpha)} \sum_{j=0}^{m-1}\left((m-j-1)^{-\alpha} Y^{\prime}\left(t_{j+1}\right)-(m-j)^{-\alpha} Y^{\prime}\left(t_{j}\right)\right)-f\left(t_{m}, Y\left(t_{m}\right)\right),
\end{aligned}
$$

after using the trapezoidal rule.

Consider the following coupled system of fractional Langevin equations:

$$
\left\{\begin{array}{l}
{ }^{c} D^{\alpha_{1}}\left({ }^{c} D^{\beta_{1}}+\frac{1}{8}\right) \psi_{1}(t)=\frac{\left|\psi_{2}(t)\right|}{7\left(1+t^{2}\right)\left(1+\left|\psi_{2}(t)\right|\right)}-\frac{\psi_{1}(t)}{5}+\ln (1+t), \quad t \in[0,1], \\
{ }^{c} D^{\alpha_{2}}\left({ }^{c} D^{\beta_{2}}+\frac{1}{7}\right) \psi_{2}(t)=\frac{\left|\psi_{1}(t)\right|}{14 \sqrt{1-t+\psi_{1}^{2}(t)}}-\frac{3 \sin \psi_{2}(t)}{5+t}-1-t^{2} .
\end{array}\right.
$$


Equipped with the nonlocal nonseparated fractional integral and fractional derivative boundary conditions,

$$
\left\{\begin{array}{l}
\psi_{1}(0)=1, \psi_{2}(0)=3, \psi_{1}^{\prime}(0)=\psi_{2}^{\prime}(0)=0, \\
\psi_{1}\left(\frac{9}{10}\right)=\frac{1}{4}\left({ }^{c} D^{1 / 4} \psi_{2}\right)\left(\frac{1}{5}\right), \\
\psi_{2}(1)=2\left(I^{5 / 4} \psi_{1}\right)\left(\frac{1}{8}\right) .
\end{array}\right.
$$

Here, $\lambda=1 / 8, k=1 / 7, a=1 / 4, b=2, a_{0}=1, b_{0}=3, p=$ $1 / 4, q=5 / 4, \xi=9 / 10, \eta=1, \mu_{1}=1 / 5, \mu_{2}=1 / 8$, and

$$
\begin{aligned}
& f\left(t, \psi_{1}, \psi_{2}\right)=\frac{\left|\psi_{2}\right|}{7\left(1+t^{2}\right)\left(1+\left|\psi_{2}\right|\right)}-\frac{\psi_{1}}{5}+\ln (1+t), \\
& g\left(t, \psi_{1}, \psi_{2}\right)=\frac{\left|\psi_{1}\right|}{14 \sqrt{1-t+\psi_{1}^{2}}}-\frac{3 \sin \psi_{2}}{5+t}-1-t^{2} .
\end{aligned}
$$

On the other part, for $(t, \zeta, \kappa),(t, \bar{\zeta}, \bar{\kappa}) \in[0,1] \times \mathbb{R} \times \mathbb{R}$, we get

$$
\begin{aligned}
|f(t, \zeta, \kappa)-f(t, \bar{\zeta}, \bar{\kappa})| & \leq \frac{1}{5}(|\zeta-\bar{\zeta}|+|\kappa-\bar{\kappa}|), \\
|g(t, \zeta, \kappa)-g(t, \bar{\zeta}, \bar{\kappa})| & \leq \frac{3}{5}(|\zeta-\bar{\zeta}|+|\kappa-\bar{\kappa}|), \\
|f(t, \zeta, \kappa)| & \leq \sigma_{0}+\frac{1}{5}|\zeta|+\frac{1}{7}|\kappa|, \\
|g(t, \zeta, \kappa)| & \leq \tau_{0}+\frac{1}{14}|\zeta|+\frac{3}{5}|\kappa|,
\end{aligned}
$$

so $\sigma_{1}=1 / 5, \sigma_{2}=1 / 7, \tau_{1}=1 / 14, \tau_{2}=3 / 5, K=1 / 5$, and $L=3 / 5$.

Case $i$. In order to illustrate Theorem 9, we take $\alpha_{1}=1 / 2, \beta_{1}=3 / 2, \alpha_{2}=3 / 4$, and $\beta_{2}=7 / 4$. Thus, $\bar{\alpha}_{1}=13 / 4$, and $\bar{\alpha}_{2}=9 / 4$. By using the Matlab program, we found that

$$
\begin{aligned}
\Delta & \simeq 0.399301327423794, \\
A_{1} & \simeq 1.171331813010056, \\
A_{2} & \simeq 0.031689241829108, \\
A_{3} & \simeq-1.262241885268691, \\
B_{1} & \simeq 1.000100634730081, \\
B_{2} & \simeq 0.002312772254050, \\
B_{3} & \simeq-2.871400693622515, \\
Q_{1} & \simeq 00.974398268143493, \\
Q_{2} & \simeq 0.043889448160305, \\
Q_{3} & \simeq 0.001217044521056, \\
Q_{4} & \simeq 0.601838570591574, \\
Q_{5} & \simeq 0.188078540898721,
\end{aligned}
$$

$$
\begin{aligned}
Q_{6} \simeq & 0.005629381698062, \\
Q_{7} \simeq & 3.713645478256922 \times 10^{-4}, \\
Q_{8} \simeq & 0.177657802566698, \\
\rho_{1} \simeq & 0.188078540898721, \\
\rho_{2} \simeq & 0.0 .177657802566698, \\
& \left(Q_{1}+Q_{3}\right) K+\left(Q_{2}+Q_{4}\right) L+\rho_{1}+\rho_{2} \\
\simeq & 0.948296217249456<1 .
\end{aligned}
$$

Thus, the hypothesis of Theorem 9 holds. Then, problems (55)-(56) have a unique solution on $[0,1]$. The behavior of the solutions $\psi_{1}(t)$ and $\psi_{2}(t)$ for Case $\mathrm{i}$ is presented in Figure 1. Table 1 displays the residuals $R_{\psi_{1}}\left(t_{m}\right)$ and $R_{\psi_{2}}\left(t_{m}\right)$ for the couple of equations given in (55) which clearly indicates the accuracy of the present algorithm.

Case ii. In order to illustrate Theorem 10, we take $\alpha_{1}=1 / 4, \beta_{1}=5 / 4, \alpha_{2}=1 / 2$, and $\beta_{2}=2$.

Thus, $\bar{\alpha}_{1}=11 / 4$ and $\bar{\alpha}_{2}=5 / 4$.

By using the Matlab program, we found that

$$
\begin{aligned}
\Delta & \simeq 0.386787685580080, \\
A_{1} & \simeq 1.140949096832007, \\
A_{2} & \simeq 0.021215616584967, \\
A_{3} & \simeq-1.201812441154092, \\
B_{1} & \simeq 1.000159825315441, \\
B_{2} & \simeq 0.004297601923856, \\
B_{3} & \simeq-2.873555329197462, \\
Q_{1} & \simeq 1.485098417429794, \\
Q_{2} & \simeq 0.056422691832900, \\
Q_{3} & \simeq 0.004245829991826, \\
Q_{4} & \simeq 0.601861587859298, \\
Q_{5} & \simeq 0.220678979659357, \\
Q_{6} & \simeq 0.003030802369281, \\
Q_{7} & \simeq 8.31262586410917 \times 10^{-4}, \\
Q_{8} & \simeq 0.142874266998083, \\
\rho_{1} & \simeq 0.220678979659357, \\
\rho_{2} & \simeq 0.142874266998083, \\
S & \simeq 0.708442401834063<1, \\
\bar{S} & \simeq 0.611596096688384<1 .
\end{aligned}
$$

Thus, all the conditions of Theorem 10 are satisfied. Then, problems (55)-(56) have one solution at the least $[0,1]$. In addition, both solutions $\psi_{1}(t)$ and $\psi_{2}(t)$ for Case ii are sketched in Figure 2. 


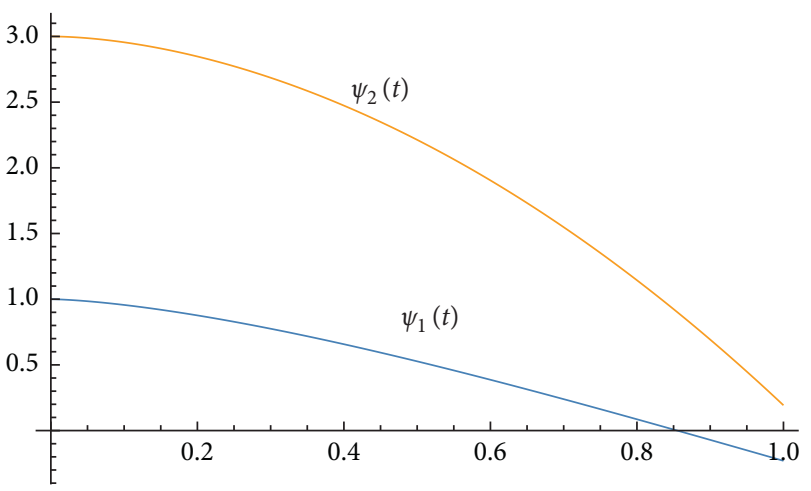

FIGURE 1: Graphs of the approximated solutions $\psi_{1}(t)$ and $\psi_{2}(t)$ for Case i.

TABLE 1: Residuals $R_{\psi_{1}}\left(t_{m}\right)$ and $R_{\psi_{2}}\left(t_{m}\right)$ for example 1 .

\begin{tabular}{ccc}
\hline$t_{m}$ & $R_{\psi_{1}}\left(t_{m}\right)$ & $R_{\psi_{2}}\left(t_{m}\right)$ \\
\hline 0.2 & $0.64265 \times 10^{-4}$ & $2.33263 \times 10^{-4}$ \\
0.2 & $3.61491 \times 10^{-3}$ & $1.51331 \times 10^{-4}$ \\
0.6 & $2.96055 \times 10^{-3}$ & $7.53023 \times 10^{-3}$ \\
0.3 & $5.12564 \times 10^{-3}$ & $2.02533 \times 10^{-3}$ \\
1.0 & $3.69052 \times 10^{-3}$ & $5.32055 \times 10^{-3}$ \\
\hline
\end{tabular}

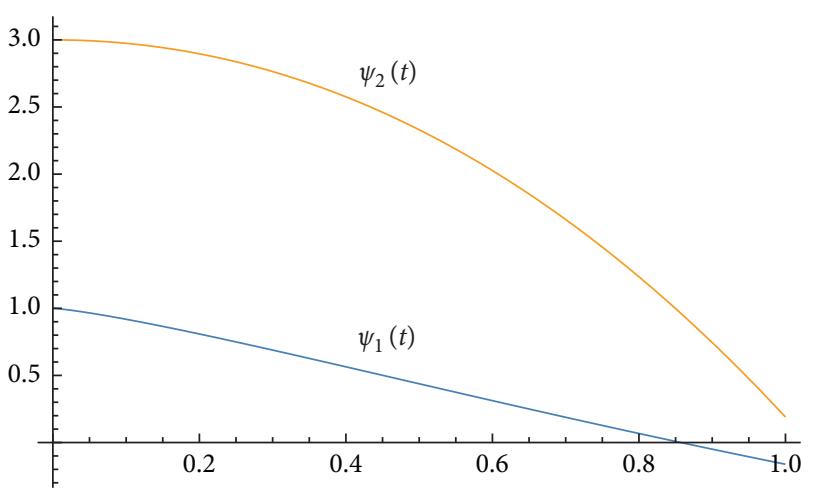

FIGURE 2: Graphs of the approximated solutions $\psi_{1}(t)$ and $\psi_{2}(t)$ for Case ii.

\section{Conclusion}

The most important features of differential equations subject to either initial or boundary conditions are the existence and uniqueness of their solutions. In this paper, we discussed the existence and uniqueness of solutions of specific type of the couple system of the Langevin differential equation in the framework of Caputo fractional derivatives and under the suzerainty of nonlocal and nonseparated boundary conditions. The boundary value problem we studied contained 6 different parameters. Because of the complexity, we were forced to use computer programs in order to find examples that would support our results. We discussed these examples from the theoretical point and solved numerically using the Adams-type predictorcorrector method by implicitly implementing the Gauss-Seidel method.

It is recommended to consider the same problem in the frame of other fractional derivatives especially the ones with no singular kernels and compare their results to the ones discussed in this paper.

\section{Data Availability}

The data used to support the findings of this study are included within the article in the references.

\section{Conflicts of Interest}

The authors declare that they have no conflicts of interest.

\section{Acknowledgments}

The authors would like to acknowledge and express their gratitude to the United Arab Emirates University, Al Ain, UAE, for providing the financial support with Grant No. 31S363-UPAR (4) 2018.

\section{References}

[1] S. G. Samko, A. A. Kilbas, and O. I. Marichev, Fractional Integrals and Derivatives: Theory and Applications, Gordon \& Breach, Yverdon, Switzerland, 1993.

[2] A. Kilbas, H. M. Srivastava, and J. J. Trujillo, Theory and Application of Fractional Differential Equations, North Holland Mathematics Studies, Elsevier, Amsterdam, Netherlands, 2006.

[3] H. Sun, Y. Zhang, D. Baleanu, W. Chen, and Y. Chen, "A new collection of real world applications of fractional calculus in science and engineering," Communications in Nonlinear Science and Numerical Simulation, vol. 64, pp. 213-231, 2018.

[4] P. Langevin, "Sur la théorie du mouvement brownien (in French) [On the theory of Brownian motion]," Comptes Rendus de l'Académie des Sciences, vol. 146, pp. 530-533, 1908.

[5] W. Coffey, Y. Kalmykov, and J. Waldron, "The Langevin equation," With applications to stochastic problems in physics, chemistry and electrical engineering, World Scientific Series in Contemporary Chemical Physics, World Scientific, vol. 14, World Scientific, River Edge, NJ, USA, 2nd edition, 2004.

[6] R. Klages, G. Radons, and M. Sokolov, Anomalous Transport: Foundations and Applications, Wiley $\mathrm{VCH}$, Weinheim, Germany, 2008.

[7] S. C. Lim, M. Li, and L. P. Teo, "Langevin equation with two fractional orders," Physics Letters A, vol. 372, no. 42, pp. 6309-6320, 2008.

[8] M. Uranagase and T. Munakata, "Generalized Langevin equation revisited: mechanical random force and self-consistent structure," Journal of Physics A: Mathematical and Theoretical, vol. 43, no. 45, p. 11, Article ID 455003, 2010.

[9] A. Lozinski, R. G. Owens, and T. N. Phillips, "The Langevin and fokker-planck equations in polymer rheology," Numerical Methods for Non-Newtonian Fluids, vol. 16, pp. 211-303, 2011.

[10] Z. Laadjal, B. Ahmed, and N. Adjeroud, "Existence and uniqueness of solutions for multi-term fractional Langevin equation with boundary conditions," Dynamics of Continuous Discrete and Impulsive Systems: Series A - Mathematical Analysis, vol. 27, no. 5, pp. 339-350, 2020.

[11] C. Torres, "Existence of solution for fractional Langevin equation: variational approach," Electronic Journal of Qualitative Theory of Differential Equations, vol. 54, no. 54, pp. 1-14, 2014. 
[12] B. Ahmad, S. K. Ntouyas, and A. Alsaedi, "On fully coupled nonlocal multi-point boundary value problems of nonlinear mixed-order fractional differential equations on an arbitrary domain," Filomat, vol. 32, no. 13, pp. 4503-4511, 2018.

[13] H. Li and J. Zhang, "Positive solutions for a system of fractional differential equations with two parameters," Journal of Function Spaces, vol. 2018, Article ID 1462505, 9 pages, 2018.

[14] S. N. Rao and M. M. Zico, "Positive solutions for a coupled system of nonlinear semipositone fractional boundary value problems," International Journal of Differential Equations, vol. 2019, Article ID 2893857, 9 pages, 2019.

[15] A. Granas and J. Dugundji, Fixed Point Theory, SpringerVerlag, New York, NY, USA, 2003.

[16] S. Abbas, M. Banerjee, and S. Momani, "Dynamical analysis of fractional-order modified logistic model," Computers \& Mathematics with Applications, vol. 62, no. 3, pp. 1098-1104, 2011.

[17] F. A. Rihan, Q. M. Al-Mdallal, H. J. AlSakaji, and A. Hashish, "A fractional-order epidemic model with time-delay and nonlinear incidence rate," Chaos, Solitons \& Fractals, vol. 126, pp. 97-105, 2019.

[18] Q. M. Al-Mdallal, M. A. Hajji, and T. Abdeljawad, "On the iterative methods for solving fractional initial value problems: new perspective," Journal of Fractional Calculus and Nonlinear Systems, vol. 2, no. 1, pp. 76-81, 2021. 